\begin{tabular}{|c|c|c|c|c|c|}
\hline MUNIBE Antropologia-Arkeologia & $n^{\circ} 66$ & $223-243$ & DONOSTIA & 2015 & ISSN 1132-2217 • eISSN 2172-4555 \\
\hline
\end{tabular}

\title{
Jefes del Sénia. Sobre la emergencia de jefaturas durante la primera Edad del Hierro en el nordeste de la Península lbérica
}

\author{
Chiefs of the Senia River. On the emergence of chiefdoms during \\ the Early Iron Age in the northeast of the Iberian Peninsula
}

PALABRAS CLAVES: Jefaturas, Protohistoria, Primer Hierro, Fenicios, Cataluña, Nordeste de la P. Ibérica.

GAKO-HITZAK: Buruzagitzak, Protohistoria, Lehen Burdina, Fenizioak, Katalunia, Iberiar Penintsularen ipar-ekialdea

KEY WORDS: Chiefdoms, Protohistory, Early Iron Age, Phoenicians, Catalonia, Northeast of the Iberian Peninsula.

David GARCIA I RUBERT(1)

\section{RESUMEN}

En los últimos años se está desarrollando un interesante y profundo debate sobre los modelos de organización política de las sociedades que habitaron en el nordeste de la Península Ibérica durante la Protohistoria. En nuestra opinión, el surgimiento de las primeras sociedades complejas en esta zona se produciría con anterioridad al desarrollo de la Cultura Ibérica. Con el objetivo de contrastarlo, presentamos un caso de estudio datado durante la primera Edad del Hierro (siglos VII-VI anE, aprox.)

\section{LABURPENA}

Azken urteotan Protohistorian Iberiar Penintsularen ipar-ekialdian bizi izan ziren gizarteen antolaketa politikoaren ereduei buruzko eztabaida interesgarri eta sakona garatzen ari da. Gure iritziz, eremu horretan lehen gizarte konplexuak Iberiar Kultura garatu aurretik sortu izango ziren. Hori kontrastatu ahal izateko, lehen Burdina Aroan datatutako (VII-VI. mendeak K.a. gutxi gorabehera) azterketa-kasu bat aurkeztu dugu.

\begin{abstract}
A discussion is being developed in recent years about the political system of the communities that inhabited the north-east of the Iberian Peninsula during the Protohistory. In this paper, we propose that the first complex societies arise in this area before the Iberian Culture. In order to contrast it, we present a case study dated in the Early Iron Age (VII-VI century BC) formed by a set of five sites located closed to each other, that we have named, as a whole, as Sant Jaume Complex. That Complex was formed by the settlements of Sant Jaume -a big and fortified house-, la Moleta del Remei -a village-, la Ferradura, el Castell -two points strategically located for the control of local communication paths- and la Cogula -perhaps a watchtower-. We follow a contextual approach, following the perspective proposed by R. D. Drennan and C. E. Peterson for the analysis and definition of the early complex societies. From this perspective, we attempt a reconstruction of Complex Sant Jaume sociopolitical organization from the specific study of a number of lines of evidence, which are interpreted in combination. The elements explored are Local Community Structure, Supra-local Community Scale, Supra-local Community Centralization, Demographic Density, Public Works Investment, Tax Rate, Conflict, Wealth and Prestige Differentiation, as well as other points as long distance Exchange or Storability of staple foods, among others. We also explore which were the sources of power that allowed the elites to control the system establishing, first, the dependency relationships and secondly, stabilizing the system. We conclude that it is possible to consider that the Sant Jaume Complex was a simple chiefdom, with a low level of development.
\end{abstract}

\section{1.- INTRODUCCIÓN}

A lo largo de los últimos años, los estudios sobre el surgimiento y la evolución de la complejidad política durante la protohistoria en el área del nordeste de la península lbérica han experimentado un notable incremento. Las causas son diversas, aunque destacan especialmente tres. En primer lugar, el aumento substancial de los trabajos de campo, sumado a la consolidación de los planteamientos pluridisciplinarios, circunstancias que han permitido multiplicar el volumen y la diversidad de la información. En se- gundo lugar, la asunción por parte de algunos investigadores de la idea de que el tipo y calidad del conocimiento actualmente disponible permiten llevar a cabo un avance significativo en la definición de los sistemas de integración sociopolítica de las comunidades protohistóricas de estos territorios. Por último, y en el caso particular de algunos de estos autores, el convencimiento de poder efectuar una lectura diferente y más completa del registro arqueológico a partir de la utilización de principios metodológicos y enfoques teóricos procedentes de la Antropología Cultural.

(1) Grup de Recerca en Arqueologia Protohistòrica (GRAP). Universidad de Barcelona. C/ Montalegre, 6, 08001 Barcelona. C/e: dgarciar@ub.edu. 
DAVID GARCIA I RUBERT

En este marco, uno de los aspectos claves estriba en la posibilidad de identificar cuándo y en función de qué parámetros se produce en este territorio el surgimiento de las primeras sociedades complejas durante la protohistoria. Asumiendo que los diversos autores que han tratado el tema lo han hecho a menudo desde posicionamientos teóricos diversos, la cual cosa genera un ruido de fondo que dificulta la realización de un análisis comparativo global para un territorio tan extenso como este -comporta, por ejemplo, tener que gestionar la utilización de categorías de idéntica denominación pero con significado diverso según el autor-, creemos poder afirmar que en el estado actual del debate no existe todavía un acuerdo al respecto. Mientras que para algunos autores se trataría de una circunstancia íntimamente ligada al desarrollo de la cultura Ibera, para otros, entre los que nos contamos, este proceso ya se habría producido, en algunas regiones, con anterioridad. La principal intención de este trabajo es precisamente contrastar esto último, y con este objetivo fijamos la atención en un caso de estudio a manera de ejemplo, el poblamiento de la región del río Sénia durante la primera Edad del Hierro. Nuestro enfoque parte, con carácter general, de formulaciones neoevolucionistas, aunque no de manera estricta ya que esta posición se combina puntualmente, como podrá comprobarse, con planteamientos propios de otras propuestas teóricas.

\section{2.- EL ESTUDIO DE LOS SISTEMAS DE ORGANI- ZACIÓN DE LAS COMUNIDADES PROTOHISTÓ- RICAS DEL NORDESTE PENINSULAR: ÚLTIMAS APORTACIONES Y NUEVAS PERSPECTIVAS}

En los últimos años se está desarrollando un interesante debate científico en relación a la identificación, definición y descripción de los sistemas de integración sociopolítica que eventualmente cabe atribuir a las sociedades de los diversos periodos protohistóricos -primera Edad del Hierro, Ibérico antiguo, pleno y final. Ss. VII-II anE- del área del nordeste de la península Ibérica. Aun sin tratarse de un tema nuevo, ya que ha sido objeto de estudio desde la primera mitad del siglo XX (SANMARTí, 2010: 91), sí que son diversas y especialmente enriquecedoras las aportaciones efectuadas recientemente.

Entre los primeros trabajos de lo que podríamos denominar etapa moderna de este tipo de enfoque conviene citar los trabajos de F. Burillo (1992-93) en relación al cambio en las formas de organización sociopolítica del poblamiento de la zona del Bajo Aragón y territorios cercanos. De manera prácticamente coetánea, para el ámbito de la zona ampurdanesa y territorios cercanos destacan igualmente los trabajos de A. Martín (1995), que analiza el desarrollo durante el Ibérico antiguo de los primeros territorios políticos de base polinuclear en la zona, centrados en el oppidum del Puig de Sant Andreu (Ullastret, Gerona). Por lo que respecta al conjunto de los territorios nororientales de Cataluña, y partiendo del análisis de los contextos funerarios, se ha propuesto así mismo la definición de una escala social de la población enterrada -incluyendo la exis- tencia de algún grupo privilegiado o élite- para cronologías de primera Edad del Hierro (PONS \& ESTEBA, 2000).

En relación al llano occidental catalán, destacan los trabajos del GIP de la Universidad de Lérida, dirigido por E. Junyent, que ha efectuado una interesante propuesta evolutiva de los sistemas de integración sociopolítica para el periodo que discurre entre los inicios del Bronce final y los primeros momentos de la iberización. Este equipo defiende, de manera genérica, el posible surgimiento de jefaturas incipientes en la región en contextos de primera Edad del Hierro, con la referencia indispensable del poblado de Vilars (Arbeca, Lérida) (GIP, 2003: 234 y 256).

En el marco de este debate, los trabajos de J. Sanmartí destacan por su carácter global tanto en términos territoriales como cronoculturales. Sus propuestas han permitido disponer de un modelo referencial que trata de explicar íntegramente los cambios sociopolíticos que se desarrollan en estos territorios a lo largo de toda la protohistoria. Desde un posicionamiento teórico que encuentra su referencia principal (SANMARTí, 2010: 91) en los modelos de cambio y evolución sociocultural elaborados por A.W. Johnson y T. Earle (2000), Sanmartí defiende que los sistemas organizativos de las comunidades protohistóricas habrían evolucionado en esta área desde formatos sociopolíticos igualitarios o pseudoigualitarios, característicos todavía de la primera Edad del Hierro (circa 700 anE), hasta modelos estatales o pseudoestatales, ya durante los últimos momentos de la cultura Ibera (circa III-II anE) (SANMARTÍ, 2004; 2010). Para este autor, por lo tanto, la génesis de la complejidad política -propiamente dicha- en este amplio territorio es un fenómeno básicamente relacionado con el desarrollo de la primera fase de la cultura lbera, de tal forma que la aparición de los primeros territorios políticos no se produciría hasta la segunda mitad del siglo VI anE (SANMARTÍ, 2004: 20). Por lo que respecta a la primera Edad del Hierro, apunta que no es posible defender el surgimiento de tipos de sociedad más complejos que aquellos de formato igualitario asociados a la figura del Big Man (SANMARTÍ, 2009: 22, 2010: 94), y que, en todo caso, la aparición de algunas primeras jefaturas incipientes se podría haber producido, en esta zona, muy hacia el final del periodo (SANMARTí et al., 2009: 229), prácticamente durante la transición al periodo ibero.

En este mismo marco de reflexión general sobre la problemática no podemos olvidar las aportaciones de G. Ruiz Zapatero, quien incidió sobre la mirada holística que conviene establecer sobre los contextos arqueológicos funerarios y los domésticos al analizar este tipo de aspectos, así como la necesidad de incorporar planteamientos que incluyan la perspectiva antropológica. Concluye este autor que el registro del nordeste de la península Ibérica indicaría el desarrollo de un proceso de diferenciación social que ya se habría iniciado a principios del Bronce final (RUIZ ZAPATERO, 2004: 326-327).

Para los territorios regados por los ríos Ebro y Sénia disponemos de las aportaciones realizadas en los últimos años por diversos equipos de investigación. Así, por ejem- 
plo, J. Diloli y su equipo (URV) proponen una posible introducción en el área del bajo Ebro, a lo largo del siglo VI anE, de concepciones cultuales de procedencia oriental, y plantean la posible relación entre este hecho y la aparición de nuevos formatos de integración política (BEA \& DILOLI, 2005). P. Moret, conjuntamente con otros autores, definió un modelo de organización social, política y económica propio de los últimos momentos de la primera Edad del Hierro y principios del Ibérico antiguo y característico de una parte de los territorios del curso bajo del río Ebro. Este modelo se fundamenta en la existencia de pequeñas residencies de tipo turriforme (casas-torre), que este autor denomina aristocráticas y que presumiblemente centralizarían el poder político en el marco de sociedades jerarquizadas de pequeño formato (MORET et al., 2006). Desde el GRAP de la Universidad de Barcelona, nuestra contribución al debate parte de un primer trabajo que propugnaba el posible surgimiento de sociedades complejas en el tránsito entre el Bronce final y la primera Edad del Hierro en algunos territorios cercanos a la desembocadura del Ebro (GARCIA i RUBERT, 2005). Posteriormente, hemos continuado participando con reflexiones de carácter más general (ARMADA et al., 2005; GARCIA i RUBERT, 2011; GARCIA i RUBERT \& MORENO, 2008; GARCIA i RUBERT et al., 2006; BEA et al., 2012). N. Rafel y su equipo, desde la Universidad de Lérida, también han reflexionado últimamente sobre esta cuestión, analizando, por un lado, las causas del cambio social y del origen del iberismo a partir del caso de estudio de la región del Matarraña-Algars (RAFEL, 2006), y explorando, por otro, en el área del curso bajo del Ebro, las posibles relaciones entre las transformaciones en arquitectura y urbanismo que se producen en la zona en el tránsito entre el Bronce final y la primera Edad del Hierro y el progresivo desarrollo de tendencias desigualitarias (RAFEL \& ARMADA, 2009).

Como últimos apuntes, y también con un carácter más global, debemos citar a J. López (2007), quien presentó un estado de la cuestión sobre los procesos de transformación sociopolítica en el área del nordeste peninsular entre el Bronce final y el Ibérico antiguo, que acompañó de algunas interesantes reflexiones generales sobre el posible desarrollo de procesos de complejización social en algunas regiones con anterioridad a la cultura Ibera. Otros autores han explorado una problemática similar desde perspectivas de análisis más particulares. Es el caso, por ejemplo, de S. Sardà, desde la óptica de las prácticas comensales (SARDÀ, 2008, 2009, 2010a, 2010b).

En resumen, comprobamos como en el marco de este debate existe un cierto consenso entre los diversos autores en considerar que al menos algunas de las sociedades que se asientan en esta área del nordeste peninsular experimentaron, durante el tránsito del Bronce final a la primera Edad del Hierro, importantes cambios por lo que respecta al formato de sus estructuras sociopolíticas. Los desacuerdos aparecen cuando se trata de determinar de manera más precisa el alcance, ritmo e intensidad de estos cambios, así como las tipologías sociopolíticas a que dan lugar. Mientras que algunos investigadores defienden que, a lo sumo, se habrían generado sociedades de tipo tribal, con el surgimiento, en algunos casos puntuales, de figuras políticas tipo Big Man, otros opinan que en algún caso ya se habrían dado pasos hacia la complejidad política y que, por lo tanto, podríamos hablar sin problemas de la aparición de las primeras jefaturas. Por nuestra parte, consideramos que numerosos indicadores señalan que en diversos puntos de este amplio territorio habrían surgido, al menos desde mediados del siglo VII anE -primera Edad del Hierro-, y quizás con anterioridad, algunas jefaturas de formato simple asociadas a auténticos territorios políticos, circunstancia que pretendemos ilustrar con un estudio de caso.

\section{3.- EL SURGIMIENTO DE LAS SOCIEDADES COMPLEJAS: DE BIG MEN Y JEFES}

En gran medida, el debate se centra en la posible existencia e identificación de indicadores arqueológicos que permitan, en el contexto apuntado, contrastar el posible desarrollo de un grado significativo de complejidad política en el tránsito entre el Bronce final y la primera Edad del Hierro. Esto ha llevado a algunos autores a explorar, desde determinados planteamientos teóricos y metodológicos, asignables muchas veces a perspectivas neoevolucionistas y a otras íntimamente relacionadas con estas, la posibilidad de identificar en el registro algunos formatos sociopolíticos propios de estos planteamientos, así como las figuras adscritas. Es por ello que resulta pertinente definir previamente qué cabe entender realmente por categorías como Big Man y Jefe en estos marcos teóricos, ya que se trata de conceptos utilizados de manera cada vez más abundante por parte de los investigadores cuando realizan este tipo de análisis.

Desde estos posicionamientos teóricos, se considera que la figura del Big Man aparece en algunas de las sociedades tradicionalmente denominadas de tipo tribal, a menudo definidas también como sociedades acéfalas. En ellas, el poder es difuso, no centralizado, y no existen liderazgos políticos adscritos ni institucionalizados. Se trata de comunidades esencialmente igualitarias por lo que se refiere al formato de su organización social, en el sentido de que ningún grupo familiar -incluyendo los de los Big Meno de otro tipo es ni política ni económicamente superior a los otros. Las unidades que conforman una sociedad de tipo tribal consisten generalmente en comunidades individuales o unidades familiares extensas, las cuales disponen de un nivel muy alto de independencia económica aun manteniéndose política e ideológicamente (incluyendo la eventual concreción física de este aspecto, como por ejemplo en el ámbito ceremonial) interdependientes. La producción se limita, en general, al ámbito de la subsistencia, con un reducido excedente que en todo caso no se centraliza al nivel de la tribu, sino al de la casa, la comunidad o la familia extensa. Este excedente, básicamente aporta seguridad en épocas de carestía, aunque pueda utilizarse puntualmente para establecer alianzas entre comunidades (CREAMER \& HAAS, 1985: 739). 
En sociedades de tipo tribal donde aparece la figura del Big Man el excedente de producción puede ser, puntualmente, generado y utilizado para obtener un cierto grado de estatus. En relación a esto conviene remarcar la idea que aunque las sociedades tribales no están en ningún caso internamente jerarquizadas, sí que pueden mantener -y esto se da de manera especial en el marco de estos sistemas de Big Man- determinados niveles de estatus de algunos individuos respecto de otros (CREAMER \& HAAS, 1985: 739). Se trata siempre de un estatus coyuntural, restringido a determinados ámbitos de las relaciones sociopolíticas (ceremonias, familia), sujeto a competencia y que queda a expensas de la capacidad de este Big Man de mantenerlo sistemáticamente mediante su capacidad de oratoria y de trabajo. En ningún caso este estatus se integra estructuralmente en roles de liderazgo, y no otorga al que lo ostenta la potestad de tomar decisiones en nombre del grupo ni de obligar a este a hacer nada, así como tampoco existe la posibilidad de transmitir este estatus por herencia. En este tipo de sociedad el poder político está, por lo tanto, muy limitado. De hecho, y en relación directa con estas limitaciones, resulta pertinente remarcar el hecho que un Big Man nunca llega al poder, sino que construye este poder (SAHLINS, 1963: 290), y lo debe hacer, además, de manera continuada. En último término, se constata habitualmente la existencia de diversos de estos Big Men en el marco de una misma comunidad tribal, compitiendo muy a menudo entre ellos por el estatus.

Por lo que respecta a las jefaturas, la definición clásica en este marco teórico apunta que se trata de organizaciones políticas de nivel intermedio, caracterizadas por la existencia de una jerarquización social marcada -con signos evidentes de las diferencias de estatus- y de una centralidad política. Desde la óptica del evolucionismo cultural, se trata propiamente de sociedades complejas. Se ha afirmado que habitualmente concentran poblaciones regionales, en las que se constata algún grado de poder adscrito e institucionalizado, rango social heredable y estratificación social (CARNEIRO, 1981; EARLE, 1987: 279, 1991: 1, 1997: 14; SERVICE, 1962: 154-55). Circunstancias estas que permiten distinguir las jefaturas de las sociedades tribales de Big Man, en las cuales, como decíamos, el liderazgo es coyuntural, no adscrito, de alcance limitado y queda íntimamente ligado al carisma y la habilidad (CHABAL et al., 2004: 27). El aspecto de las dimensiones demográficas mínimas consideradas necesarias para el desarrollo de un sistema de jefatura ha sido revisado significativamente a la baja de manera reciente, al haber sido posible validar la existencia de jefaturas simples formadas por grupos de pocos centenares de personas (DRENNAN \& PETERSON, 2006: 3963; PETERSON et al., 2010: 5757 y 5759, fig. 7; PETERSON \& LU, 2013).

Las jefaturas han sido también definidas como entidades políticas centralizadas de carácter multinuclear -o supralocales-, compuestas por comunidades locales interdependientes económicamente que están bajo el control permanente de un jefe. Caracterizadas, por lo tanto, por la existencia de dos niveles de organización política, el de los asentamientos y el superior -que organiza estos asentamientos- (CARNEIRO, 1981: 45-46, 2002: 90), orden que no se constata en los sistemas tribales, incluyendo los que incorporan la figura del Big Man. En un principio, fueron definidas también como sociedades redistributivas (SAHLINS, 1958; RENFREW, 1976: 171-173; SERVICE, 1962: 144). Con todo, conviene resaltar las críticas que la vocación universal de esta atribución ha recibido (CARNEIRO, 1981: 60-61) y los ejemplos diversos que ilustran como en muchas jefaturas esta dinámica de colecta y redistribución de la producción regional, en realidad, no está presente (STEPONAITIS, 1978: 420). Este componente redistributivo de algunas jefaturas, cuando se documenta, ha de ser considerado más como una posible consecuencia que como una causa primaria e ineludible de su desarrollo (CREAMER \& HAAS, 1985: 740).

En último término, existe un acuerdo cada vez más general en considerar que resulta conveniente no utilizar el término como una categoría tipológica cerrada. Detrás de la etiqueta única de "jefatura" se esconden una gran diversidad de formatos y de grados diferentes de desigualdad institucionalizada (EARLE, 1991: 2; FEINMAN, 2005) que en muchos casos presentan niveles de complejidad, características, formas de organizar las relaciones sociales, demografía, extensión territorial, magnitudes e, incluso, causas y patrones de desarrollo, muy diferentes entre sí (ATHENS, 1979: 25; CARNEIRO, 1981: 46; EARLE, 1991: 2; FEINMAN, 1991: 230; DRENNAN \& PETERSON, 2006: 3960 y 3966, 2011: 64). EI concepto, por lo tanto, da cobijo a numerosas variantes que van desde las jefaturas más simples a sociedades con una elevada complejidad y jerarquización interna (JOHNSON \& EARLE, 2000: 245).

El término concreto, en tanto que denominación de un tipo evolutivo dotado durante los años 60 del siglo XX de una serie de atributos determinados $-y$, de hecho, todo el conjunto de las propuestas neoevolucionistas- recibió con posterioridad a su definición una intensa crítica (SPENCER, 1990; CHABAL et al., 2004: 27). Estas circunstancias llevaron incluso a numerosos investigadores a considerar improductivo tratar de identificar en el registro arqueológico los atributos anteriormente apuntados con el objetivo de clasificar una eventual sociedad del pasado dentro de este tipo (EARLE, 1987: 280). Con todo, numerosos autores han seguido defendiendo la validez general de las propuestas neoevolucionistas, aunque apuntando la necesidad de reconsiderar algunos de sus postulados a la luz de las críticas, así como también de asumir que cualquier reflexión respecto a la tipología sociopolítica de una comunidad debería partir siempre de la consideración de los factores históricos y medioambientales específicos (SPENCER, 1990: 23), es decir, del análisis contextual. Personalmente, consideramos que algunas de las categorías utilizadas en este marco teórico, y especialmente las relacionadas con las tipologías sociopolíticas, pueden ser útiles -una vez valoradas convenientemente las críticas realizadas- para el análisis arqueológico. 
La gran diversidad de modelos de organización sociopolítica de tipo jefatura existente obliga a los investigadores a realizar un importante esfuerzo suplementario en términos comparativos. Desde una perspectiva antropológica, estos casos de estudio resultan interesantes independientemente de su caracterización cronocultural y de su localización geográfica. Cada estudio de caso particular se acaba sumando a la multiplicidad de ejemplos ya conocidos, permitiendo de este modo que del análisis comparativo surja un conocimiento cada vez más preciso de las causas y mecanismos relacionados con el desarrollo de las sociedades complejas tempranas. Con todo, para que ello sea realmente útil desde una perspectiva que se propone sea universal, se requiere que cada caso de estudio sea descrito y analizado siguiendo pautas, criterios, categorías, terminología y orientaciones similares, única forma viable de que este tipo de aproximaciones comparativas sea efectivo. En relación a ello, R. D. Drennan y C. E. Peterson, siguiendo otros trabajos previos, propusieron una estrategia básica de aproximación a una parte de esta problemática, con el objetivo de superar el desafío especifico que supone el estudio comparativo de las sociedades complejas tempranas (DRENNAN \& PETERSON, 2011). Una propuesta que adoptamos en este trabajo y que, aunque no suponga exactamente una novedad absoluta, sí que pensamos que contribuye a la necesaria sistematización de este tipo de estudios y, por lo tanto, a hacerlos más útiles y efectivos. También ha de permitir que el caso particular que aquí presentamos encuentre fácil acomodo conceptual y terminológico en el marco de los estudios de estos procesos desarrollados desde una perspectiva antropológica, y que por lo tanto contribuya al mejor conocimiento de la problemática a una escala universal. Esencialmente, la propuesta se fundamenta en la exploración particular de una serie de ítems considerados determinantes en el aspecto a tratar. Entre ellos, se propone la exploración de la estructura propia de las comunidades locales ${ }^{1}$ en la región objeto de estudio, la existencia o no de una escala comunitaria de tipo supralocal, la posibilidad de identificar, o no, una centralización comunitaria de ámbito supralocal, la posibilidad de identificar la realización de inversiones en obras que puedan ser calificadas de una u otra forma como públicas/comunitarias, la posible contribución en forma de tributos, la existencia o no de conflictos y el estudio de sus características y significación real, y la posibilidad de identificar diferencias en términos de riqueza, ritualidad, prestigio y productividad, entre otros.

Cada una de estas categorías son analizadas desde una perspectiva contextual, y se intenta definir de la manera más precisa posible sus características y su importancia relativa respecto del contexto local, supralocal -si es el caso- y regional. El orden en que hemos abordado y detallado estos ítems en nuestro trabajo no presupone ninguna direccionalidad en el análisis, ni tampoco otorga un diferencial de peso relativo. Tampoco cabe considerar estas categorías de análisis como circunstancias todas ellas propias exclusivamente de las sociedades de tipo complejo, ya que en determinadas circunstancias y dependiendo del formato que adopten, algunas de ellas pueden ser identificadas también en sociedades de tipo igualitario. De hecho, es el contexto y la manera como se presentan y eventualmente se utilizan lo que ha de permitir determinar, en cada caso, si alguna o algunas de estas categorías sugiere -o incluso certifica- por ella misma, en base al planteamiento teórico propuesto, la existencia de la complejidad política, pudiéndose dar la circunstancia, además, de que para cada caso de estudio un eventual papel determinante en este aspecto pueda recaer en categorías diferentes.

Obviamente, debe tenerse igualmente presente la innegable interrelación que, con todo, mantienen entre sí las diversas categorías de análisis propuestas. Será precisamente la puesta en valoración conjunta de los resultados alcanzados en la interpretación de cada categoría lo que permitirá, finalmente, representar -hasta donde ello sea posible- el sistema de integración sociopolítica de la comunidad objeto de estudio.

\section{4.- EL POBLAMIENTO DE LA REGIÓN DEL SÉNIA DURANTE LA PRIMERA EDAD DEL HIE- RRO. ESTRUCTURA BÁSICA DE LAS COMUNI- DADES LOCALES}

Desde este planteamiento teórico pretendemos analizar a manera de ejemplo una parte del poblamiento protohistórico de la región del río Sénia, un territorio de casi $1.000 \mathrm{~km}^{2}$ situado una veintena de kilómetros al sur de la desembocadura del río Ebro, a caballo entre Cataluña y el País Valenciano. Los límites geográficos de esta región son la costa mediterránea al este, las sierras de Irta, Solá, Perdiguera y Garrotxa al sur, los Ports de Beseit, la Sierra del Turmell y las Montañas de Benifassá al oeste y las Sierras de Godall y de Montsiá al norte (fig. 1). El llano litoral de Vinaroz-Benicarló, de formación cuaternaria y surcado por diversos cursos de agua de caudal muy débil, ocupa buena parte de la región. Las comunicaciones con los territorios limítrofes son fáciles y ágiles, gracias a una serie de anchos corredores naturales -depresiones de Alcalá, La Galera, Ulldecona y Les Coves-, orientados en sentido noreste/suroeste y dispuestos entre las sierras mencionadas.

Como ya hemos apuntado en trabajos anteriores (GARCIA i RUBERT, 2011), a partir aproximadamente de

\footnotetext{
${ }^{1}$ El término "comunidad" puede servir para denominar diferentes realidades y tipos de agrupaciones humanas (PETERSON \& DRENNAN, 2005: 5), al tratarse de una expresión de tipo genérico. En este trabajo denominamos "comunidad local" a la "comunidad" concentrada en un solo núcleo de poblamiento, independientemente de su tamaño y funcionalidad, adoptando así la nomenclatura propia de múltiples estudios antropológicos (p.e.: PETERSON et al., 2010). El concepto "comunidad supralocal" define aquí un conjunto de comunidades locales que mantienen un nivel de organización sociopolítica que abarca e integra todos los núcleos. No confundir estos términos con el concepto "grupo local", uno de los cuatro niveles básicos de integración política definidos por Earle (1994: 944-945), relacionable en gran medida con el concepto tribal de Service (1962), y con el cual este autor define la agrupación de diversas familias en un único asentamiento, asumiendo la ausencia de cualquier tipo de poder adscrito o institucionalizado.
} 


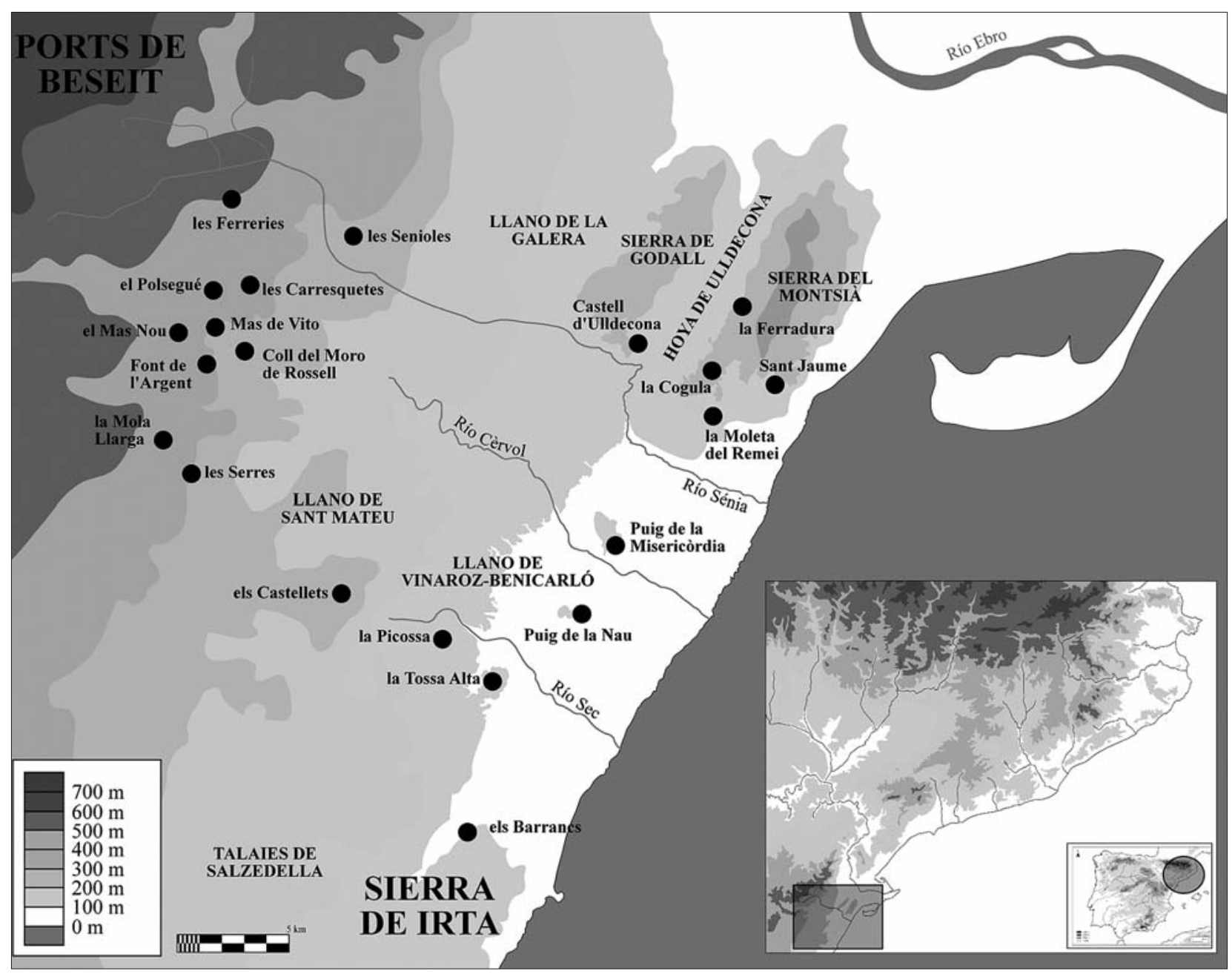

Fig. 1. Asentamientos localizados en las tierras del río Sénia durante el primer hierro (@ GRAP-UB). / Settlements located in the lands of the Sénia river during the early Iron Age.

mediados del siglo VII anE, y en el marco cronocultural de los inicios de la primera Edad del Hierro, documentamos en esta región la implantación de una alta densidad de asentamientos, ex-novo en su práctica totalidad. Se trata siempre de comunidades locales muy nucleizadas, consistentes en agrupaciones residenciales (asentamientos) muy compactas y separadas las unas de las otras por una extensión variable de terreno sin evidencias de ocupación. Estos asentamientos, habitualmente situados en altura, se caracterizan también por disponer de un número diverso de ámbitos, construidos en gran medida con materiales no perecederos, adosados los unos a los otros compartiendo paredes medianeras y protegidos conjuntamente o bien por un muro de cierre o bien por una muralla.

\section{5.- EXPLORACIÓN DE LA ESCALA COMUNITA- RIA SUPRALOCAL}

A partir de esta estructura formal básica de las comunidades locales, la apertura del foco a una escala regional con el objetivo de analizar el mapa de distribución de los puntos de hábitat sugiere la existencia de un cierto patrón general. Este se caracteriza, entre otros aspectos, por la aparente definición de diversas agrupaciones de núcleos, separadas entre sí por extensas áreas sin ocupación. En su momento sugerimos, aunque sin profundizar en ello, que estas agrupaciones podrían resultar significativas en términos sociopolíticos, y que cada conjunto podría ser el resultado de la existencia de una profunda interrelación entre las comunidades locales que los componen. Es decir, que lejos de tratarse de núcleos políticamente independientes, podríamos estar ante diversas comunidades supralocales.

Es, precisamente, para tratar de corroborar esta hipótesis que centramos nuestra atención en una de estas agrupaciones, la que en ya su momento denominamos Complejo Sant Jaume (CSJ) (GARCIA i RUBERT, 2011) (fig. 2), y que hasta el momento hemos explorado desde otras perspectivas. Se trata de una agrupación de asentamientos situada en el extremo nororiental de esta región, formada por un conjunto de cinco comunidades locales, con características formales muy diferentes entre sí pero con una ocu- 


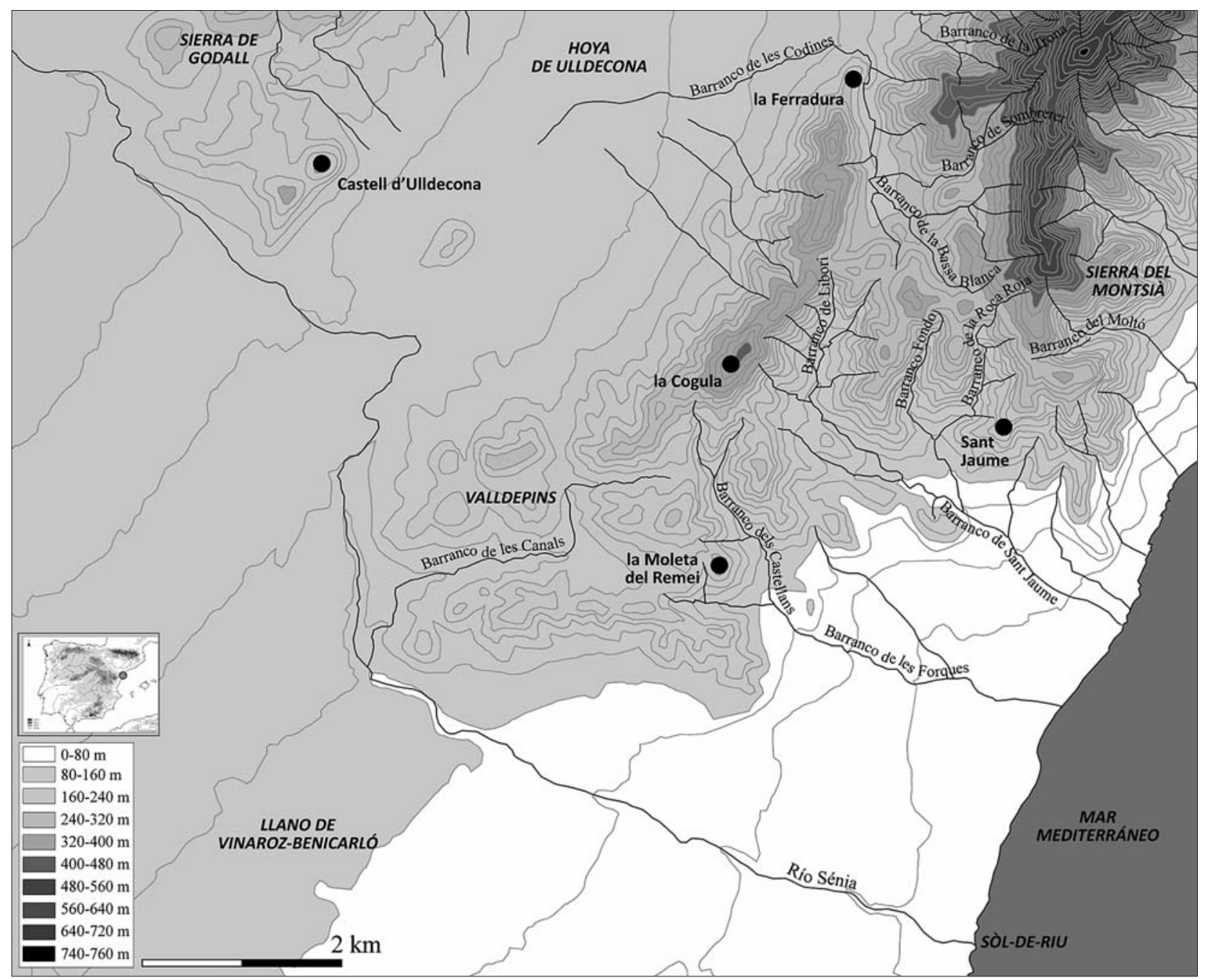

Fig. 2. Comunidades locales que conforman el Complejo Sant Jaume (CSJ) (@ GRAP-UB). / Complex Sant Jaume local communities.

pación coetánea durante la primera Edad del Hierro: Moleta del Remei y Sant Jaume (en Alcanar) y Ferradura, Cogula y Castell (en Ulldecona). Si bien en su momento ya planteamos la posible existencia de algún tipo de interrelación entre ellos, a la vista, sobretodo, de aspectos básicos como la extrema proximidad que mantienen, el diferencial de sus dimensiones y la posibilidad de atribuirles funcionalidades específicas y aparentemente complementarias (GARCIA i RUBERT, 2005; 2011), no habíamos abordado todavía el estudio profundo tanto de la naturaleza de estas posibles relaciones como de las características del sistema sociopolítico que eventualmente sería propio de la agrupación. Por lo que respecta a las características específicas de cada núcleo y a su estudio tipológico-funcional nos remitimos a los diversos estudios ya publicados, algunos de los cuales incorporan, además, visiones de conjunto y listados bibliográficos (GARCIA i RUBERT, 2011).

Estos estudios previos han permitido avanzar notablemente -aunque con diferencias, ciertamente, por lo que respecta a la profundidad y al grado de certeza de las propuestas- en la interpretación funcional de cada uno de estos cinco asentamientos. Esto ha podido ser establecido a partir de los análisis a escala micro y semimicro, los cuales han permitido comprobar que, como pasa en otros múltiples ejemplos, la variabilidad en los atributos de determinados núcleos habitados contemporáneos y situados muy cercanos entre sí refleja en realidad las diferencias en su funcionalidad respectiva (ATHENS, 1979: 114). Desde este punto de vista, el listado de los tipos funcionales definidos en los estudios previos realizados incluiría un par de posibles puntos de control territorial local -Ferradura y Castell-, una atalaya con vocación de control regional -Cogula, un poblado de dimensiones notables para este contexto cronocultural, ya no solo a una escala local sino también regional -Moleta del Remei- y una gran casa compleja y fortificada -Sant Jaume-. La suma de las poblaciones de estos núcleos no debió de superar los 500 habitantes.

Definidos los componentes de la agrupación de núcleos y referenciadas sus características básicas, la primera pregunta que debemos hacernos es si estuvieron 
realmente interrelacionados sociopolíticamente. En el caso de poder confirmar esta circunstancia, el siguiente objetivo consistiría en tratar de concretar la naturaleza, características e implicaciones de estas relaciones. Conviene apuntar, previamente, que el hecho de poder validar eventualmente la existencia de una interacción entre núcleos de hábitat cercanos no tiene por que implicar necesariamente que estas relaciones se traduzcan en un sistema sociopolítico de tipo complejo. Con todo, resulta igualmente cierto que no podremos pasar a valorar la posible existencia tanto de relaciones de dependencia como de una jerarquización entre los núcleos de una agrupación determinada -es decir, la existencia de una entidad que podamos considerar propiamente como una comunidad supralocal- si, previamente, no hemos podido validar -o, al menos, inferir con suficiente solvencia- que la profunda interrelación entre comunidades locales que este tipo de sociedades complejas tienen siempre como fundamento se produjo realmente en nuestro caso (PETERSON y DRENNAN, 2005: 6).

Son diversos los elementos que pueden ayudarnos a explorar la existencia o no de una interrelación significativa entre los asentamientos que conforman el CSJ. Buena parte de ellos derivan de las características de su patrón de asentamiento, ya detalladas en su momento (GARCIA i RUBERT, 2011). Sintetizando, este patrón se caracteriza por una selección de puntos de ocupación que se localizan de manera extremadamente cerca los unos de los otros. Estos núcleos son erigidos en cimas con alturas absolutas y relativas discretas, se sitúan muy cercanos a la costa y aparecen asociados a excelentes vías de comunicación, a puntos de obtención de agua y a buenas tierras agrícolas. Solamente uno de ellos, La Cogula, no responde íntegramente a este patrón: su altura absoluta -que duplica la del resto de núcleos- sugiere el ejercicio de una funcionalidad estratégica singular. La localización de algunos de los otros núcleos más pequeños, como La Ferradura o El Castell, sugiere igualmente la voluntad de ejercer el control de diversas rutas de comunicación.

La proximidad es un factor clave al explorar el grado de interacción que eventualmente podría haber existido entre dos o más asentamientos. En el caso que aquí planteamos resulta especialmente significativo: la máxima distancia entre núcleos se da entre Castell y Sant Jaume $(7,50$ km) y la mínima entre La Cogula y La Moleta $(2,10 \mathrm{~km})$; el resto de valores se mantiene en todos los casos por debajo de los 5,50 km de separación, con una media para el conjunto de 4,224 km. Esta extrema proximidad -y la consiguiente superposición masiva de las teóricas áreas de captación respectivas- es posiblemente el factor que de manera más intensa induce a pensar en la necesaria existencia de una interrelación entre los núcleos. La ausencia de tensiones, en relación a la captación de recursos, entre núcleos tan extremadamente cercanos entre sí debe ser interpretada como un indicio de la existencia de un marco de interrelación política, de carácter pacífico, que habría hecho posible la coexistencia a medio/largo término en un área geográficamente tan reducida.
Resulta igualmente pertinente apuntar la -comparativamente- notable distancia existente entre este conjunto de asentamientos y los otros más cercanos, el Puig de la Misericordia de Vinaroz y el Puig de la Nau de Benicarló, situados a 10 y $12 \mathrm{~km}$ respectivamente. Resulta lógico pensar, visto el gran espacio intermedio sin evidencias de poblamiento, que el grado de interrelación que debieron mantener entre sí los asentamientos agrupados al norte del Sénia debió ser substancialmente mayor que el que podrían haber mantenido estos mismos núcleos con los de Vinaroz y Benicarló, así como con otros todavía más alejados.

Otros criterios permiten profundizar todavía más en esta misma línea. Las funcionalidades atribuidas a cada núcleo a partir de los estudios contextuales admiten, cuando se valoran en conjunto, una significativa lectura en clave de complementariedad. Adoptando una perspectiva global, observamos como los asentamientos más pequeños se sitúan en la periferia de este territorio, y todos ellos se relacionan funcionalmente con el control estratégico del área y de las rutas locales y regionales. Parecen proteger el gran poblado y la gran casa fortificada -situados en un sector más resguardado-, al controlar los accesos y el territorio a diversas escalas. Se da lo que parece un juego de equilibrios y de complementariedades funcionales, visualizado especialmente en la cuidada geolocalización y en el formato de cada núcleo, que, combinados con la extrema proximidad que estos mantienen, difícilmente puede explicarse sin la existencia de un grado de interrelación e incluso de coordinación política muy elevado.

Por otro lado, existen diversos indicadores que a una escala micro revelan un comportamiento diferencial de algunos elementos del registro entre lo que constatamos, en términos similares, en el conjunto de estos asentamientos y lo que observamos, en cambio, en el resto de núcleos más alejados. Aunque no es el único, el ejemplo más significativo es, en este sentido, el de la cerámica fenicia, al tratarse del resultado de una circunstancia tan importante en este marco como son los intercambios comerciales a larga distancia. En los yacimientos que aquí tratamos, la cerámica fenicia mantiene una presencia muy elevada, llegando a representar hasta un $11 \%$ del total de fragmentos cerámicos en Moleta, un 25\% en La Ferradura y un 30\% en Sant Jaume (GARCIA i RUBERT et al., 2004). En otros asentamientos cercanos, pero fuera ya de esta agrupación, estos porcentajes disminuyen de forma significativa. En el Puig de la Nau, por ejemplo, situado a 12 km de distancia, estos materiales representan ya, tan solo, un 1,16\% del total (OLIVER y GUSI, 1995: 124, cuadro 2). Y todo ello, aunque este último yacimiento se encuentra situado en un punto tan cercano a la línea de costa como lo está Sant Jaume. Estos datos no tan solo señalan la probable existencia de contactos comerciales directos y privilegiados entre alguno de estos núcleos y los navegantes fenicios sino que, por lo que a este apartado nos interesa, indican también la existencia de una interdependencia económica fluida, estable y intensa entre estos asentamientos, suficiente como para que el resultado de estas transacciones circulara de manera normalizada entre ellos. A diferencia, 
por otro lado, de lo que sucede con el resto de núcleos de la región, donde la llegada de estos productos tuvo un carácter aparentemente mucho más restringido.

En definitiva, el análisis combinado del patrón de asentamiento y del registro arqueológico pone de manifiesto la existencia de una intensa interrelación entre estos cinco núcleos, de naturaleza sociopolítica y pacífica. Más allá de esta idea básica, algunos de los indicios igualmente apuntados permitirían incluso proponer la existencia de un nivel de organización que integrara las múltiples comunidades locales, circunstancia que por ella misma ya supondría un indicador de complejidad política.

\section{6.- INDICIOS DE UNA CENTRALIDAD DE ÁM- BITO SUPRALOCAL}

La existencia de una jerarquía entre los componentes de un grupo de asentamientos sociopolíticamente interrelacionados es admitido, generalmente, como un signo propio de sociedades complejas (JOHNSON, 1977) y, de manera más específica, como uno de los indicadores claves de las jefaturas (EARLE, 1987: 289). En el análisis arqueológico, esta jerarquización queda especialmente evidenciada cuando podemos identificar el ejercicio de las funciones de centro político por parte de un núcleo determinado. Determinar la existencia de relaciones de dependencia dentro de un conjunto de núcleos, la naturaleza de estas relaciones y, eventualmente, definir un orden jerárquico no representan, con todo, cuestiones triviales. Tan solo resulta posible inferirlas con posterioridad a la definición precisa del patrón de asentamiento, de la tipología de cada núcleo, de la función o funciones que les podamos atribuir y de las actividades diferenciales que, eventualmente, pudieran llevar a cabo los habitantes respectivos. Es la realización de un análisis comparativo que permita señalar diferencias significativas en estos parámetros lo que puede hacer posible identificar diferencias de tipo sociopolítico entre dos núcleos cercanos.

Con respecto a los núcleos de los cuales conocemos suficientemente su planteamiento arquitectónico, estos pueden dividirse tipológicamente en dos grupos. Por un lado, aquellos que en su formulación planimétrica responden a modelos muy conocidos en el ámbito local y regional y que hunden sus raíces en momentos antiguos del Bronce final. Por otro, aquellos cuya planta resulta una absoluta novedad no ya tan solo a nivel regional sino, incluso, a una escala peninsular. En el primer grupo incluiríamos la Moleta y la Ferradura y, en el segundo, Sant Jaume (fig. 3).

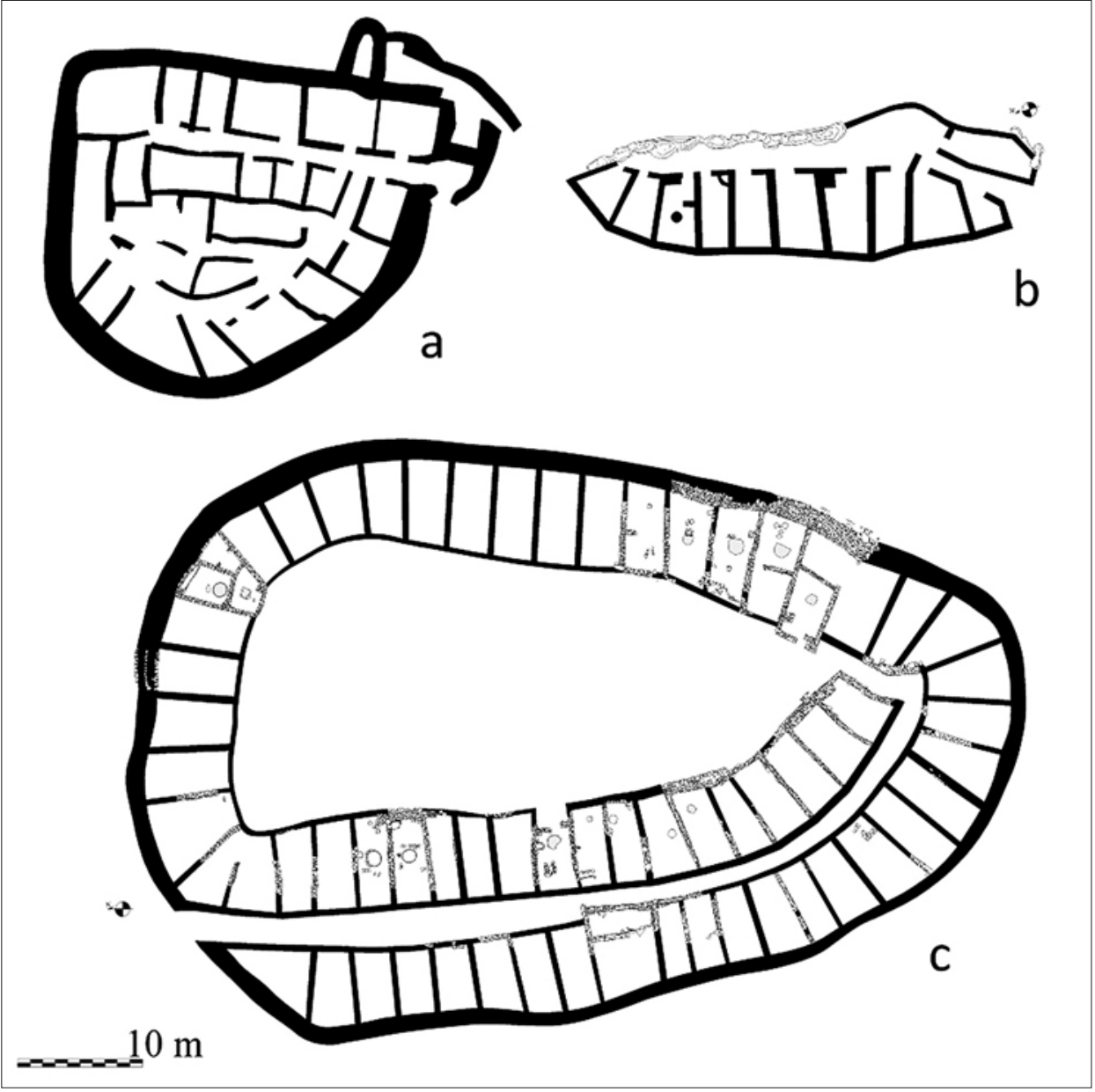

Fig. 3. Croquis de los principales asentamientos del CSJ: a) Sant Jaume, b) Ferradura, c) Moleta (@) GRAP-UB). / Sketch of the main CSJ settlements:a) Sant Jaume, b) Ferradura, c) Moleta del Remei. 
Cada uno de estos dos formatos parece evocar una concepción sociológica igualmente diversa, que de hecho parece encontrar posteriormente su correlación en el análisis de los registros respectivos. La Moleta (fig. 4), poblado de plaza central, residencia de un conjunto comunitario amplio y plurifamiliar, transmite de forma intensa, a través del formato -similar y homogéneo- y disposición de los diversos espacios de hábitat (GARCIA i RUBERT, 2004), la idea del valor de la comunidad (RUIZ ZAPATERO, 2001: 283), emanada de un ambiente formal propio de una aparente isonomía social (MAYA, 1993: 16). Algo similar puede decirse, a otra escala, de La Ferradura (fig. 5). Sant Jaume,

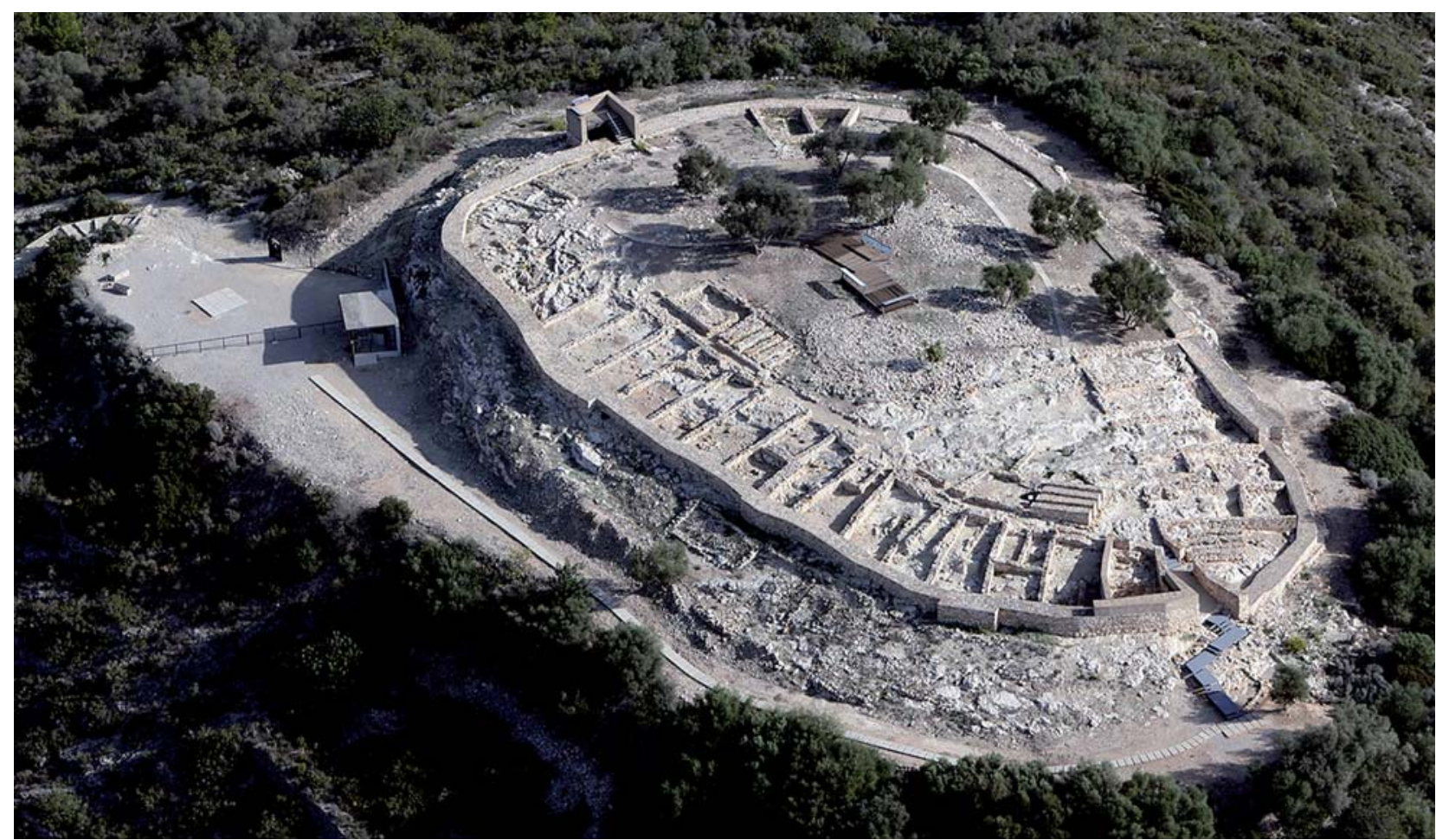

Fig. 4. Poblado de La Moleta del Remei (@ GRAP-UB). / Moleta del Remei settlement.

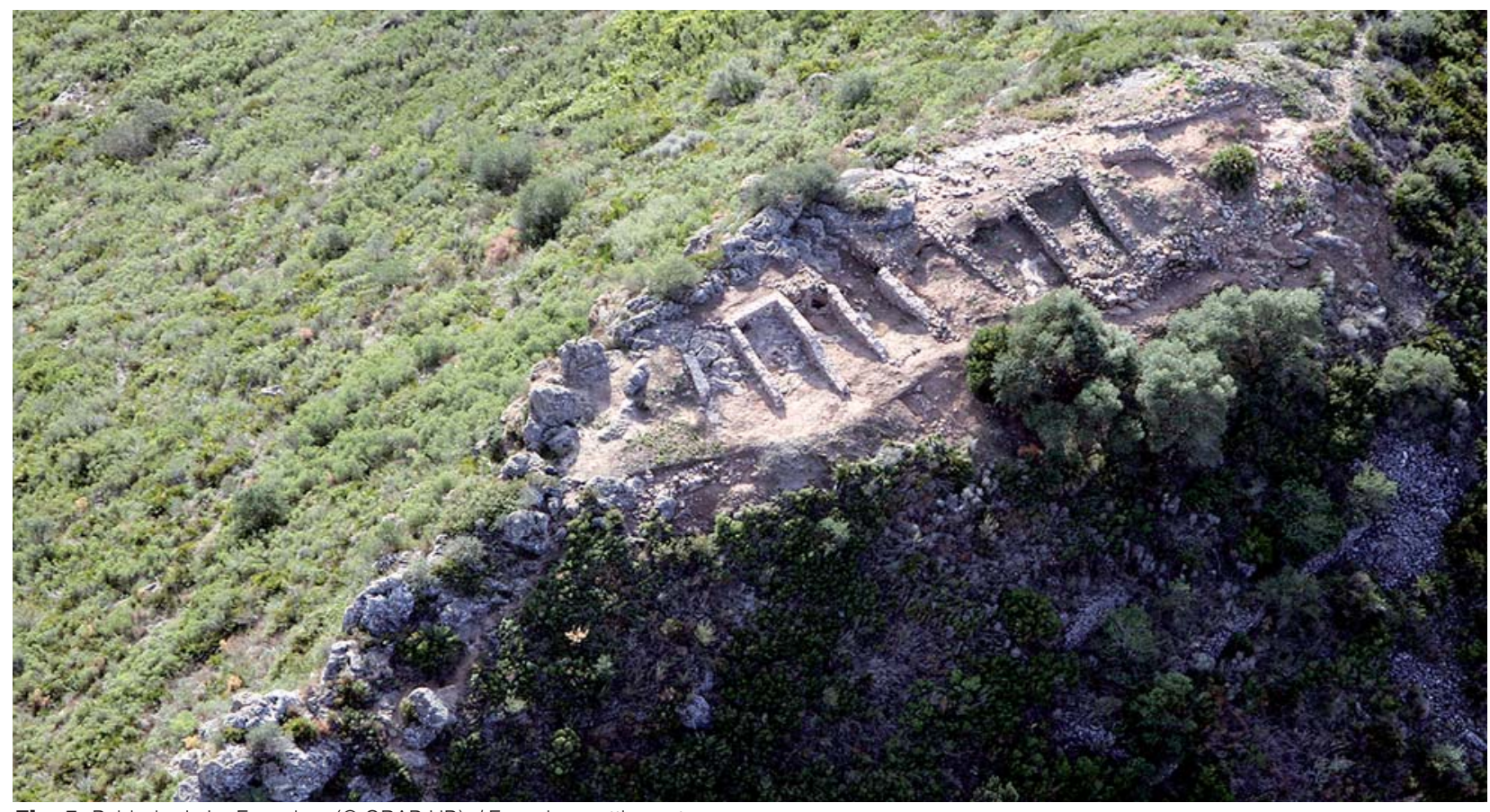

Fig. 5. Poblado de La Ferradura (C GRAP-UB). / Ferradura settlement. 
Las viviendas de la Moleta presentan una superficie media de $21 \mathrm{~m}^{2}$, lo que significa que los residentes en Sant Jaume $\left(700 \mathrm{~m}^{2}\right)$ disponían de casi 30 veces más de su- en cambio, es, en su conjunto, una gran casa, compleja, aislada, fortificada y de aspecto singular (GARCIA i RUBERT, 2009) (fig. 6 y 7).
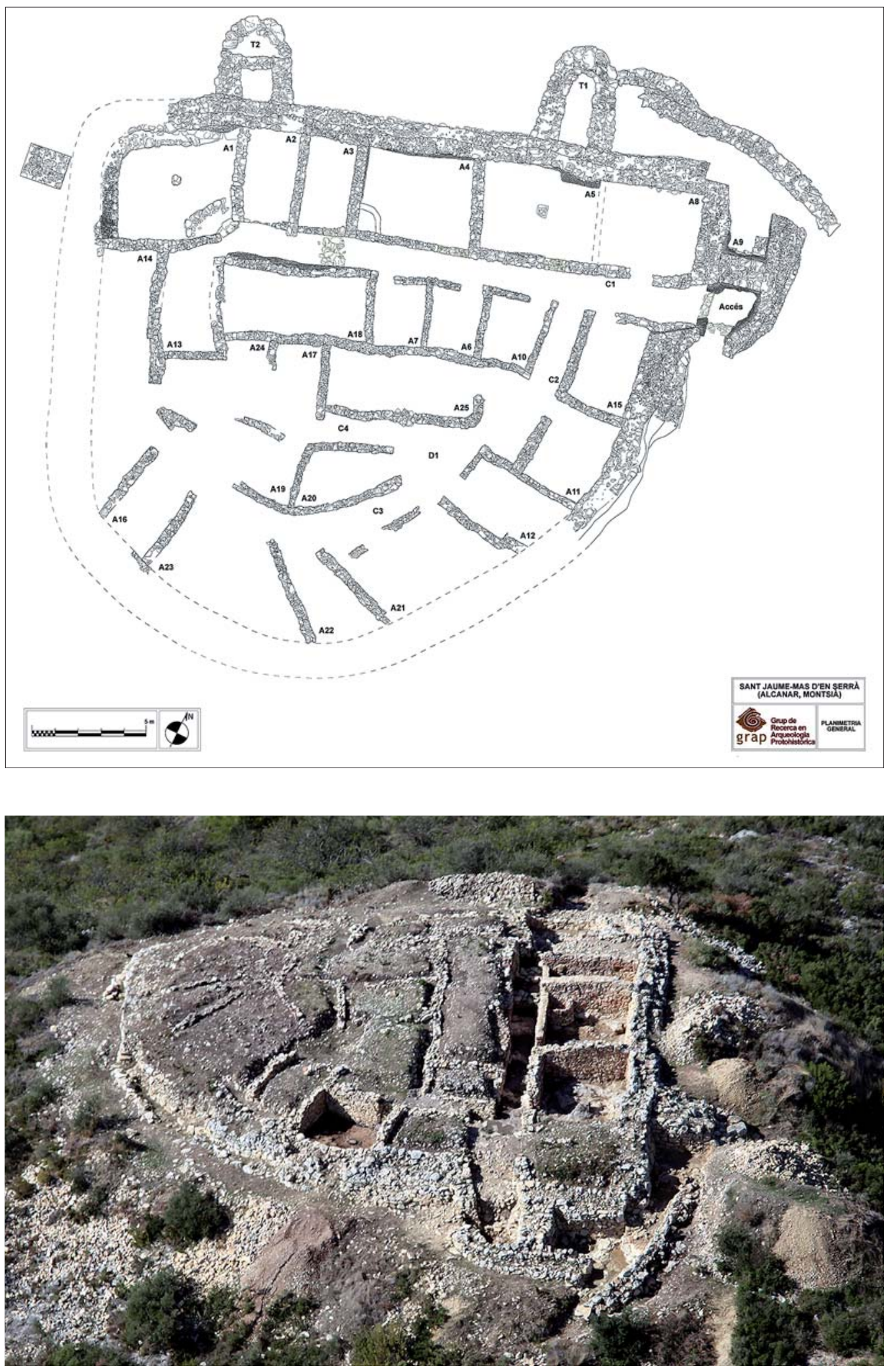

Fig. 6. Asentamiento de Sant Jaume (C GRAP-UB). I Sant Jaume settlement.
Fig. 7. Asentamiento de Sant Jaume (@ GRAP-UB). / Sant Jaume settlement. 
perficie total de espacio doméstico. Como ya hemos descrito en trabajos anteriores, los dos modelos de vivienda también se diferencian por el tipo de construcción, por el planteamiento arquitectónico, por la distribución interna y por la relación que mantienen con otras residencias. Mientras que la casa común se plantea mayoritariamente con el formato de sala única, sin divisiones internas, Sant Jaume no tan solo está multicompartimentada sino que se organiza internamente en áreas funcionalmente diferenciadas. A lo cual cabe añadir las estructuras defensivas, inexistentes en Ferradura y Moleta al nivel de las casas -y reducidas a una muralla simple al nivel del conjunto del poblado- mientras que Sant Jaume incorpora múltiples y potentes elementos defensivos. Comprobamos también como mientras los habitantes de Moleta -y también los de la Ferradura- han de cohabitar en el contexto de un mismo espacio comunitario, hasta el punto que cada casa comparte paredes medianeras con las dos que tiene a los lados, Sant Jaume adopta una localización aislada, manteniéndose separada del resto de viviendas.

Conviene no olvidar que la estructura arquitectónica de las viviendas es a menudo un símbolo visible de las diferencias en riquezas materiales (HENDERSON \& OSTLER, 2005: 13-16). En este sentido, a nuestro entender las diferencias aquí constatadas entre los dos tipos de residencia revelan, también, la existencia de diferencias significativas de estatus entre los habitantes de los diversos núcleos: el derecho de ocupar una vivienda de las dimensiones y características de Sant Jaume ha de fundamentarse necesariamente en la adscripción a un nivel sociopolítico diferenciado.
En otro orden de cosas, pero explorando igualmente la eventual existencia de diferencias de estatus y de estándar de vida entre los habitantes de uno y otro asentamiento, constatamos como el registro de Sant Jaume indica que determinadas actividades que cabe catalogar en primera instancia como de tipo comercial y social se llevaban a término en este asentamiento con una vocación eminentemente política, circunstancia que no observamos en el resto de núcleos, o al menos no de forma tan rotunda y numerosa. A manera de ejemplo, comprobamos como la cerámica fenicia tiene una presencia especialmente importante, tanto en términos de cantidad como de calidad y diversidad (GARCIA i RUBERT et al., 2004), y como ha sido posible documentar incluso la existencia en su interior de un almacén repleto de ánforas vinarias (fig. 8) Ilegadas al yacimiento como resultado de este comercio (GARCIA i RUBERT \& MORENO, 2008), resultando estas dos circunstancias absolutamente singulares tanto a una escala local como regional. Dada la extrema proximidad del yacimiento a la línea de costa, entendemos que los residentes participarían en redes extralocales, gracias a las cuales tendrían un acceso privilegiado a lo que cabe definir como bienes de valor exóticos llegados por vía marítima, y entre ellos muy especialmente al vino fenicio. Intuimos, con todo, que la imbricación de Sant Jaume en estas redes extralocales fue todavía mucho más compleja, ya que debió incluir también relaciones con otras comunidades supralocales regionales más o menos cercanas. Estas resultarían imprescindibles, por ejemplo, para el aprovisionamiento del metal que sería necesario aportar como contrapartida en las transacciones comerciales desarro-

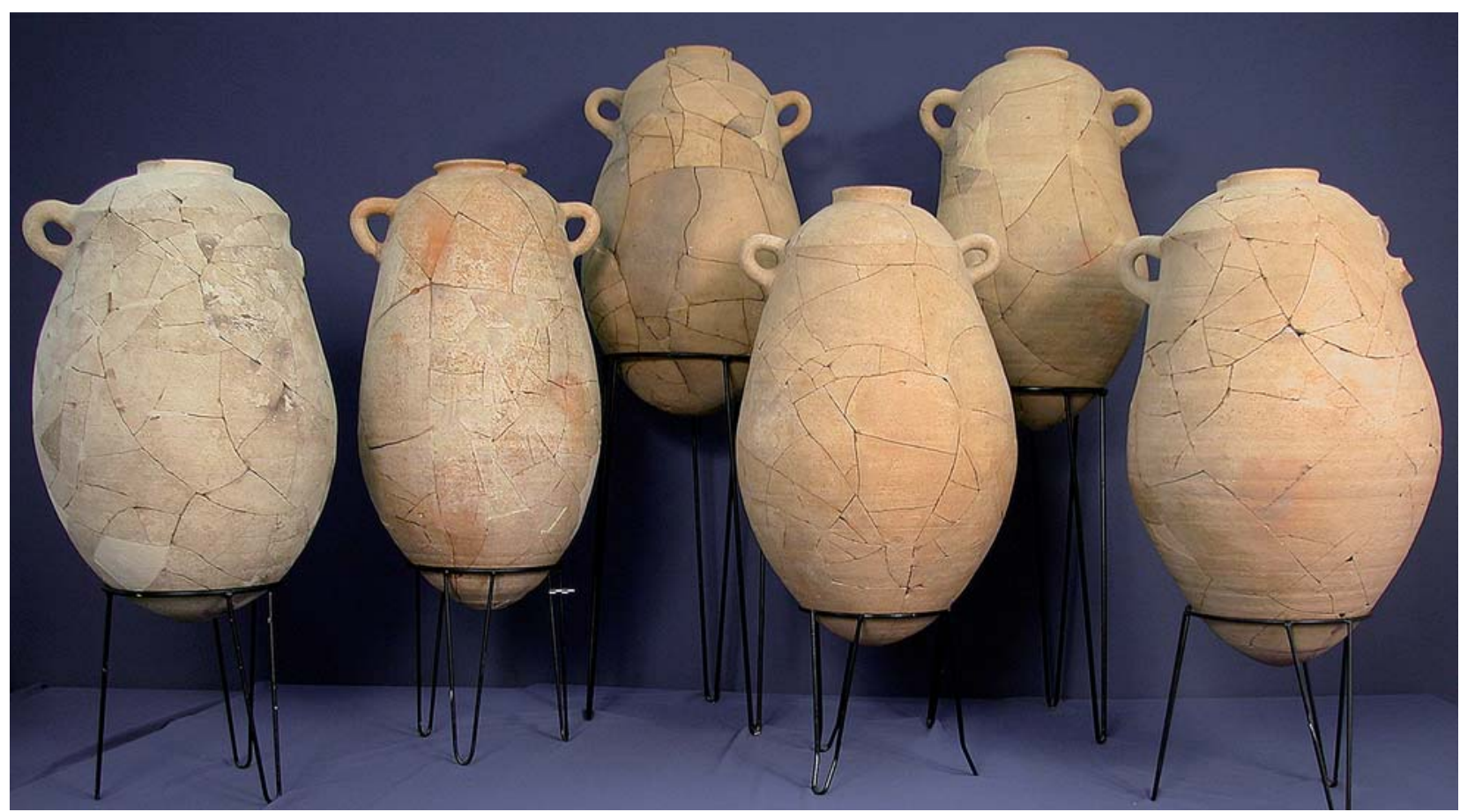

Fig. 8. Ánforas fenicias T.10.1.2.1 recuperadas en Sant Jaume (@GRAP-UB. Restauración: Sra. Isabel Moreno). / T.10.1.2.1 Phoenician amphoras recovered in Sant Jaume (Restoration: Mrs. Isabel Moreno). 
lladas con los fenicios. La existencia de relaciones entre estos asentamientos y otras comunidades del entorno queda en cierta manera también evidenciada, de manera secundaria, por la presencia de cerámica fenicia -aunque sea con niveles muy discretos- en todos los asentamientos coetáneos identificados en la región del Sénia, muy probablemente procedente de Sant Jaume y obtenida en el marco de dinámicas redistributivas.

El vino fenicio, que fue claramente el elemento más utilizado como bien de prestigio en esta zona a causa de su exotismo, de sus valores organolépticos, de su escasez y de la aparente incapacidad de las sociedades indígenas locales para producirlo, presenta una característica particular, ausente en otros bienes similares: es un producto perecedero. Si bien, como es sabido, los bienes de prestigio entran a menudo en una dinámica de intercambio que los puede llevar a circular durante mucho tiempo, pasando de mano en mano (SIKLÓSI, 2004: 8), algunos, como el vino, tienen necesariamente un recorrido espacial y temporal corto y deben consumirse antes de que se estropeen. Como consecuencia de ello, los personajes que disponían de un acceso a este producto y pretendían hacer de él un uso político debieron tender a hacerlo circular y a consumirlo de manera rápida. El interés de estas elites en utilizarlo en el marco de una economía política llevaría, por tanto, a los interesados a estimular y generar la celebración de ceremonias de banquete, de tipología, orientaciones y magnitudes diversas -dependiendo del perfil de los comensales-, al ser este uno de los contextos de donde se podría obtener un mejor rendimiento político a un bien de prestigio de este tipo. Por lo que respecta a los asentamientos del curso bajo del Sénia, es precisamente en Sant Jaume donde ha sido posible documentar la celebración de banquetes, a partir de diversos indicadores arqueológicos que incluyen conjuntos singulares de vajilla (fig. 9), elementos de metal relacionados con el tratamiento de la comida y de la bebida, conjuntos numerosos de ánforas vinarias, el almacenaje de una gran cantidad de productos comestibles, cuadras donde estabular animales -algunos de los cuales estarían probablemente destinados a ser consumidos en este contexto- e, incluso, el propio espacio físico (ámbito A1) que probablemente fue destinado a la celebración de estas ceremonias (Font et al., 2014; GARCIA i RUBERT \& MORENO, 2009; SARDÀ, 2009).

En definitiva, el carácter singular de los productos fenicios -especialmente el vino-, la manera de obtenerlos, el tipo de redistribución que se hacía, su presencia abundante en Sant Jaume y el probable consumo de parte de ellos en el marco de determinados banquetes singulares y/o restringidos son circunstancias que parecen indicar que su uso debe entenderse incardinado en el marco de una economía política. El acceso privilegiado a los bienes de prestigio de origen fenicio que, a la luz del registro, se tenía desde Sant Jaume y el uso que de ellos se hacía permite interpretar estos elementos en clave de fuente de poder. Por lo que respecta al conjunto de asentamientos aquí estudiados, este tipo de dinámica se identifica de manera exclusiva en este núcleo.
Todo lo que hemos visto hasta ahora parecería indicar, por lo tanto, que en el marco de este conjunto de asentamientos es efectivamente posible identificar la existencia de un centro político, situado en Sant Jaume. Hemos podido comprobar como, en tanto que residencia, Sant Jaume es muy diferente del resto de casas localizadas en las comunidades locales cercanas, tanto por dimensiones como por tipo constructivo y por localización, tres circunstancias que caracterizan, a menudo, la forma como los jefes singularizan su residencia (EARLE, 1987: 291). En realidad, y como testimonian numerosos ejemplos alrededor del mundo (PETERSON et al., 2010: 5765), la simple identificación de una residencia compleja ya resulta habitualmente un indicador suficiente de la actividad política de las élites, al punto que la construcción de residencias diferenciales para los líderes constituye una manifestación arqueológica clásica de la desigualdad social institucionalizada (DRENNAN et al., 2010: 45). Incluso en algunos estudios antropológicos, en que grandes residencias habían sido inicialmente asociadas a formatos políticos de carácter más igualitario y a la figura del Big Man, esta adscripción ha sido posteriormente revisada, toda vez que un análisis más profundo ha permitido captar su más que probable adscripción, en realidad, a la figura de los jefes. Uno de los casos más evidentes es, en este sentido, las "substantial houses full of weighty possessions" de las comunidades de indios pescadores de la costa noroeste de Norteamérica (NETTING, 1977; JOHNSON \& EARLE, 2000: 212-215). Los datos presentados hasta aquí parecen indicar que, de manera similar a lo que ha sido documentado antropológicamente en otras latitudes y momentos históricos (HENDERSON \& OSTLER, 2005: 16), y especialmente en momentos de emergencia de la estratificación (EARLE, 1997:44), la élite política residente en Sant Jaume había obtenido ya la capacidad de construir y conservar su casa como lugar central en el contexto general de la comunidad supralocal. Más aun: que la ambivalencia funcional inherente al edificio -sede política y, a la vez, casa adscrita a un grupo familiar concreto- convertía de facto esta residencia en un recurso material que se podía heredar y también, muy posiblemente, en una construcción altamente simbólica desde una perspectiva política.

Circunstancias similares han sido identificadas en algunas sociedades de jefatura, en las que la casa diferenciada del líder acaba convirtiéndose, también, en una fuente de autoridad política individual (HENDERSON y OSTLER, 2005: 13-16). Ello parece depender, en último término, de la habilidad del líder y, especialmente, de la capacidad de este para conseguir interrelacionar conceptualmente su casa, singular ya de por sí por el simple hecho de ser más grande y de quedar aislada, con algunas dinámicas que de manera recurrente se llevan a cabo en su interior o bien se gestionan desde ella y que bajo determinadas circunstancias puedan acabar siendo consideradas esenciales por parte del conjunto de la comunidad. En el caso de Sant Jaume, pensamos que esto podría haberse producido -y hablaremos de ello de forma más detallada en el siguiente apartado- si, 


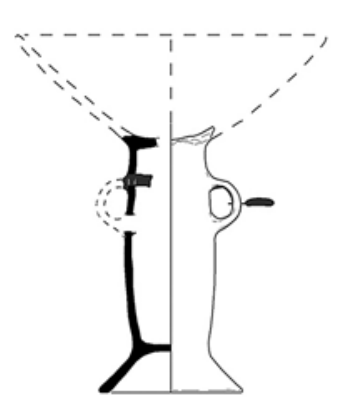

दो
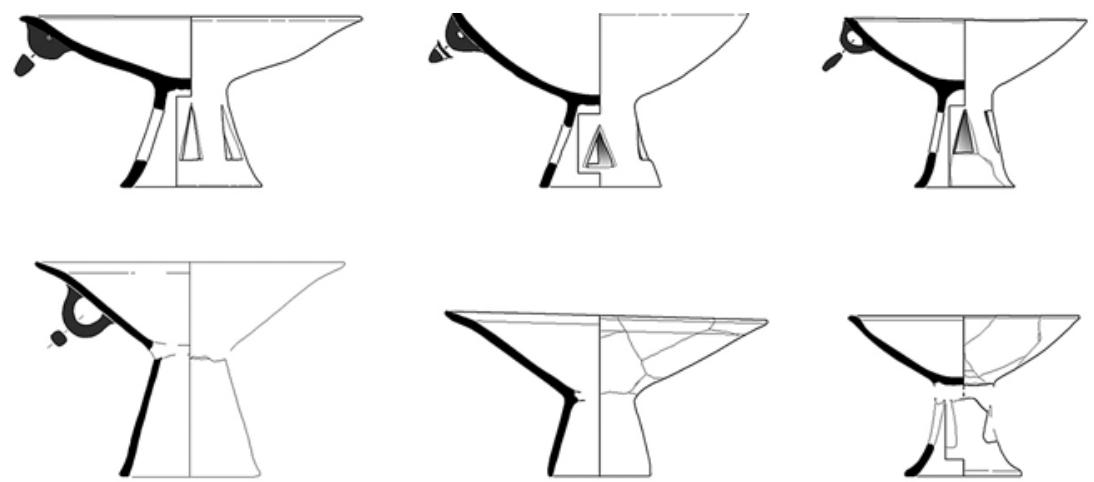

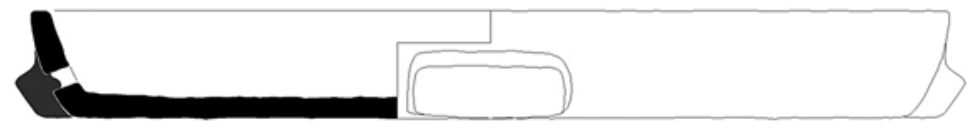

$\longrightarrow$
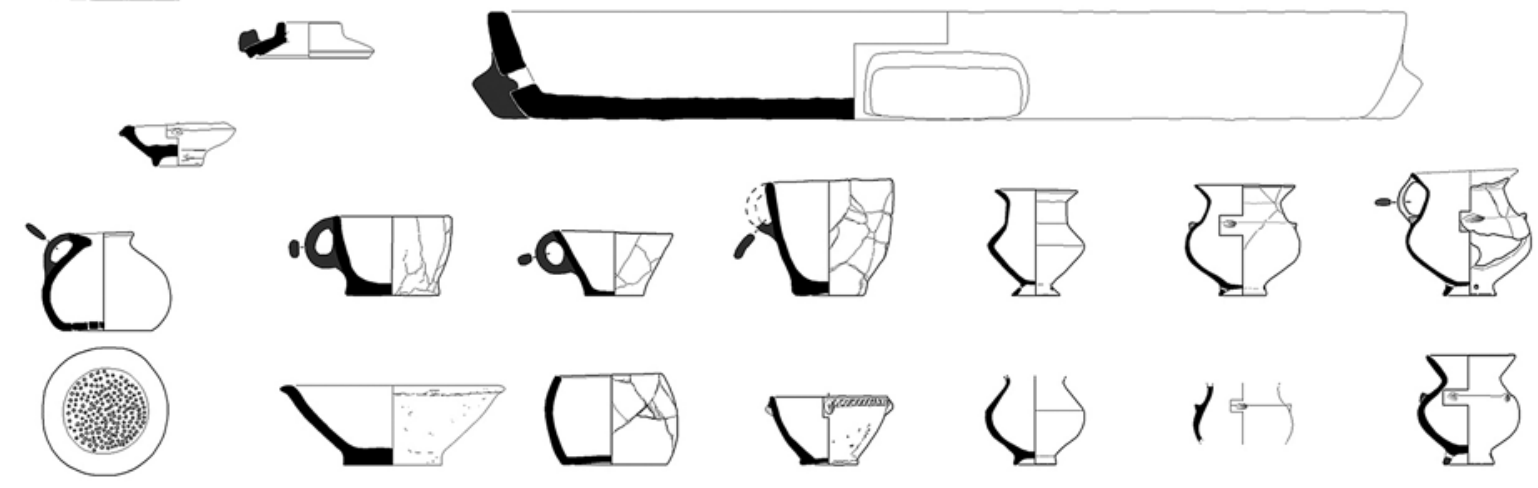

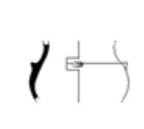
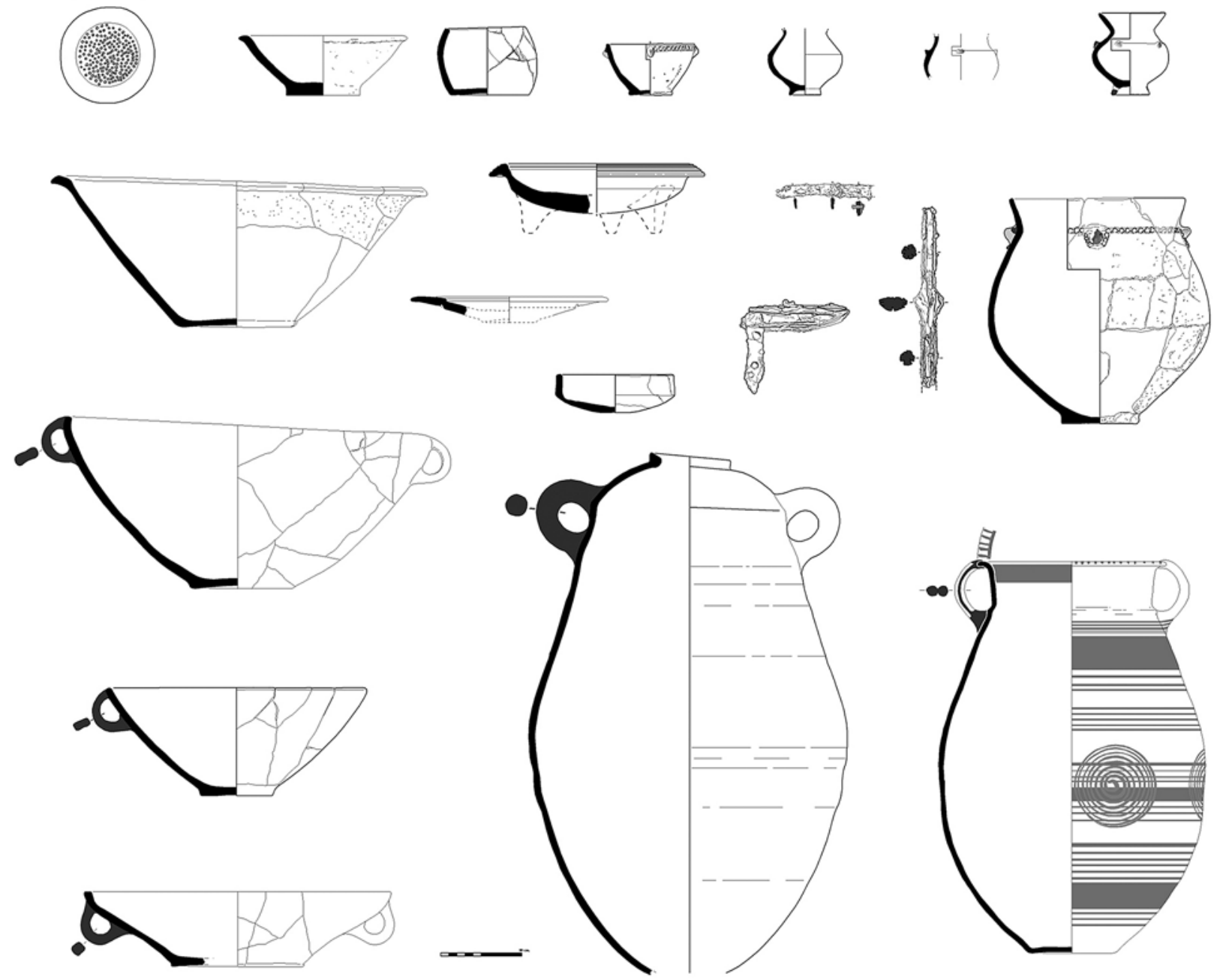

Fig. 9. Servicio de vajilla recuperado en Sant Jaume (@ GRAP-UB). / Set of ceramic and iron tableware recovered from Sant Jaume. 
como sospechamos, se hubiera dado el caso que la residencia se hubiera acabado convirtiendo -o bien ya hubiera sido conceptualizada así desde un principio- en una suerte de símbolo de las relaciones comerciales con el mundo fenicio. Como derivada de este hecho, el edificio podría haber simbolizado también otra serie de aspectos íntimamente relacionados, como por ejemplo la capacidad de sus residentes de garantizar la llegada de bienes de valor exóticos a la región de manera estable y recurrente y, en consecuencia, la posibilidad que la comunidad accediera, gracias a las gestiones desarrolladas desde esta casa, al menos a una parte de estos bienes. De hecho, la más que probable existencia de un acceso diferencial a un tipo de recurso exótico que debe cualificarse como de altamente estratégico en este contexto, es uno de los elementos que de manera más determinante debió permitir a los habitantes de Sant Jaume condicionar e incluso dirigir una parte de las acciones de los habitantes del resto de núcleos.

Todas las circunstancias apuntadas podrían haber acabado revistiendo el edificio del aura necesaria para poder convertirse en esa importante fuente de autoridad de que hablábamos. Sant Jaume podría representar, en este sentido, un ejemplo de cómo las élites pueden llegar a disponer de la habilidad y la capacidad no tan solo de conseguir que el conjunto de la comunidad supralocal les construya una casa de características diferenciales, sino también de conseguir que esta residencia se convierta en un símbolo de carácter múltiple altamente valorado por el grupo, con el objetivo puesto siempre en reafirmar su posición privilegiada y reproducirla. De esta forma, la residencia se convierte, al menos potencialmente, en el lugar de concentración y perpetuación del poder político (HENDERSON y OSTLER, 2005: 13-16), quedando asociada a la institucionalización del poder que caracterizaba la sociedad a nivel estructural y reafirmando todavía más si cabe, con su sola presencia, la solidez -real o aparente- del sistema. La propia existencia de Sant Jaume en tanto que casa, sus connotaciones de lugar central de esta comunidad supralocal, el estatus elevado que disfrutan sus residentes y el resto de circunstancias que la rodean nos están indicando, en definitiva, que no estamos ante liderazgos de tipo temporal o coyuntural, sino de carácter estructural y con una clara vocación de perdurar, circunstancias que hemos de considerar, en gran medida, asumidas por el conjunto del grupo.

En resumen, con el análisis realizado en los dos últimos apartados, que ha permitido constatar la existencia de una fuerte interacción de tipo sociopolítico entre los núcleos e identificar una centralidad política, se dan los condicionantes básicos que permiten la identificación de una comunidad supralocal. Todo parece indicar, además, que esta comunidad habría desarrollado un tipo de liderazgo adscrito de carácter estructural y formas institucionalizadas de desigualdad, constatándose también la existencia de al menos dos niveles en la jerarquía de los núcleos.

\section{7.- INVERSIONES EN CONSTRUCCIONES PÚ- BLICAS}

En función de lo expuesto hasta el momento y de los estudios realizados, del análisis del registro arqueológico se infiere que el tipo de organización sociopolítica que caracteriza este conjunto de núcleos implicó, entre otros aspectos, la utilización del trabajo del grupo por parte de la élite con el objetivo, al menos, de disponer de una residencia de grandes dimensiones y físicamente separada de las del resto de la comunidad supralocal. Este comportamiento ha sido identificado de manera similar en muchos otros casos alrededor del mundo (EARLE, 1997: 44, entre muchos otros). Como en esos otros ejemplos, resulta evidente que por sus dimensiones y complejidad, Sant Jaume no pudo ser erigido tan solo por sus futuros residentes, sino que fue necesariamente una parte importante de la propia comunidad supralocal, habitantes en su mayoría del resto de núcleos, quien llevó a término su construcción. Este hecho tiene unas importantes implicaciones sociopolíticas, ya que si bien el desarrollo de trabajos comunitarios no constituye por sí solo un indicador inflexible de complejidad social, el hecho de que el resultado de este esfuerzo común quede en manos privadas sí que resulta un indicador más de la existencia de una jerarquía sociopolítica (ATHENS, 1979: 141). Además, resulta indudable que el tipo de obra realizado implica la necesaria existencia de un grado notable de organización centralizada del trabajo que garantizase el éxito del proyecto, circunstancia que en este caso pensamos que denota el ejercicio de un grado considerable de autoridad y reafirma la idea de la existencia de una notable jerarquización.

La construcción de elaboradas residencias para los dirigentes por parte de la comunidad supralocal se incluye entre los trabajos comunitarios propios de muchos ejemplos de jefatura alrededor del mundo, debido a la conexión obvia de estos trabajos con la voluntad y el control político de las élites (PETERSON \& DRENNAN, 2011: 123). Con todo, no olvidemos que el caso concreto de Sant Jaume, que puede y debe analizarse también desde este punto de vista, presenta connotaciones muy particulares, ya que se trata de un caso único: carece de paralelos formales, precedentes o coetáneos, en su ámbito regional. A parte del hecho de que el edificio sea una obra singular en términos arquitectónicos y de diseño, igual o más innovador y transcendente resulta la circunstancia de que por primera vez en esta área del nordeste peninsular -al menos, en función de los datos actualmente disponibles- un grupo familiar proponga -y consiga- vivir en una casa de mayores dimensiones y situada al margen del resto del grupo. Es por ese hecho, novedoso en este contexto cronocultural, y por otra serie de razones asociadas que no resultaría en nada contradictorio con lo que hemos dicho hasta ahora considerar, igualmente, que ante estas circunstancias resultara necesario generar, en el preciso momento de construcción del edificio e inmediatamente 
antes, determinadas muletas argumentales añadidas que facilitasen la aceptación por parte del grupo del nuevo estado de cosas, quizás nunca planteado con anterioridad. Especialmente, en relación a la contundente escenificación del diferencial social que se estaba generando con la propia construcción del edificio, y a lo que el tipo y localización de la nueva vivienda implicaba respecto del estatus de los futuros residentes. En este sentido, la aceptación de estas importantes novedades por parte de la comunidad podría ser el resultado, quizás, de la percepción de esta construcción singular por parte del grupo como un elemento orientado a incrementar de alguna forma el bienestar general de toda la comunidad fuera esta una circunstancia real o bien simplemente el resultado de una hábil manipulación ideológica-, más allá de estar destinada, también, a ejercer las funciones de residencia de un grupo familiar en particular. Este eventual incremento del bienestar comunitario que vendría a garantizar/simbolizar el nuevo edificio podría ser de tipo económico (¿ligazón inseparable del edificio con el proceso de obtención, almacenaje y redistribución de productos exóticos llegados a través del comercio con el mundo fenicio?), simbólico, religioso o incluso ser el resultado de una combinación de estos y otros aspectos.

Por lo tanto, si aceptásemos la posible asunción por parte del grupo de un argumentario de este tipo, el cual permitiera entender el edificio, también, como algo con un interés especial para el conjunto del cuerpo social, ello justificaría plenamente que incluyéramos su construcción dentro de los trabajos comunitarios denominados "construcciones públicas". Y recordemos que la construcción de proyectos enmarcados en el ámbito de los trabajos públicos, los cuales exceden de la capacidad de un único grupo familiar, representa un rasgo arqueológico clásico de las jefaturas, precisamente a causa de que el volumen de esfuerzo laboral que es necesario invertir requiere, entre otros aspectos, de unos importantes esfuerzos organizativos por parte de las elites que lo deben gestionar (DRENNAN \& PETERSON, 2011: 74). Este tipo de trabajos públicos son habitualmente considerados como la materialización del poder político y social, independientemente del formato con que se presenten -ya sea a partir de la construcción de elementos monumentales o bien de elementos más utilitarios como fortalezas, grandes residencias, murallas o infraestructura agrícola, en tanto que responden siempre a las directrices de las élites (PETERSON \& DRENNAN, 2011: 122).

En definitiva, entendemos que el edificio de Sant Jaume podría haber tenido múltiples lecturas, pudiendo haber sido considerado por el conjunto de esta comunidad supralocal no tan solo como la gran residencia del linaje o parte del linaje imperante y como sede política central sino, también, y como resultado de una importante y hábil manipulación ideológica, como un edificio destinado a cumplir una función considerada especialmente importante para el grupo. Sin descartar que pudiera haber estado revestido, incluso, de un cierto carácter monumental.

\section{8.- CONFLICTO}

Un posible indicador igualmente valorable es la presencia/ausencia de armas, así como su significado en el contexto. Tradicionalmente, algunos arqueólogos peninsulares han considerado imprescindible, para poder aceptar la existencia de una jerarquía institucionalizada, la constatación de la presencia de armas en el registro. Con todo, existen alrededor del mundo múltiples ejemplos de jefaturas en las cuales no están presentes ni la iconografía de la guerra ni las armas, y en las que tampoco se documentan estructuras defensivas o fortificaciones asociadas a los asentamientos, porqué las fuentes de poder son otras y se localizan en otros contextos. Un buen ejemplo de ello son las pequeñas jefaturas de la fase Hongshan de la región china de Chifeng (DRENNAN \& PETERSON, 2006: 3965; PETERSON y LU, 2013), entre muchos otros. Numerosas aproximaciones antropológicas muestran, incluso, que, a veces, la importancia de estas armas -cuando efectivamente existen- es más que relativa por lo que se refiere a su relación con la estructura sociopolítica de la comunidad que las utiliza.

Dicho esto, resulta igualmente cierto que la eventual identificación del conflicto y de las armas en un contexto arqueológico determinado puede ser clave, en determinadas circunstancias, para el proceso de definición del modelo sociopolítico propio de la comunidad que las utilizó. Y no olvidemos tampoco que, efectivamente, el conflicto puede ser utilizado también como otra fuente de poder en algunos sistemas de jefatura. Como sucede a menudo, la clave radica en la realización de un correcto análisis contextual. En el caso que nos ocupa, el conflicto está claramente presente, como parecen mostrar las notables preocupaciones defensivas -con diferencias claras- de los asentamientos y la propia destrucción violenta y global de todos los núcleos del CSJ, circunstancia esta última que debe interpretarse desde nuestro punto de vista como una agresión externa que afectó al conjunto de esta comunidad supralocal. Las armas utilizadas en este conflicto fueron, mayoritariamente, el arco y la honda. En mucha menor medida, podrían haberse utilizado lanzas con punta de bronce, como indicarían los dos ejemplares localizados -descontextualizados- en la Moleta del Remei, y que pueden adscribirse tipológicamente a las producciones típicas del Bronce final y de la primera Edad del Hierro de la zona (QUESADA, 1997: 344, fig. 195). Todo ello, sin menospreciar la posible utilización de lanzas u otros elementos realizados exclusivamente con materiales perecederos, que en todo caso no se han conservado. Además de las armas más comunes, debemos valorar la posibilidad que algunos de los objetos singulares recuperados en Sant Jaume hayan sido también utilizados como tales, cumpliendo a la vez la función de arma y de bien de prestigio. Es el caso de una hacha o azuela de hierro, tipológica y técnicamente singular a una escala regional, y de la cual tan solo se conoce otro ejemplar en Cataluña, recuperado en el poblado del Coll del Moro de Serra d'Almos (Tivisa, Tarragona) y al que se le atribuye una cronología más tardía, de segunda mitad del siglo VI anE (CELA et al., 1999: 107). 
Aunque los datos indican, pues, la existencia de situaciones de conflicto -que en último término se manifiestan de manera dramática para el conjunto de los asentamientos- y la existencia de armas -en algún caso puntual, incluso de posible carácter singular-, los datos resultan todavía demasiado escasos como para poder afirmar con rotundidad que el conflicto fue utilizado, en este marco, como una fuente de poder por parte de las élites. Tan solo la potente y singular fortificación de Sant Jaume sugiere de manera más destacada esta circunstancia, al estar localizada, precisamente, en lo que interpretamos como una gran residencia singular, y al verse reforzada su potencia semiótica por el tremendo contraste que supone la ausencia de este tipo de elementos defensivos en el resto de núcleos, a una escala incluso regional. Con todo, deberemos esperar a recabar más datos para poder confirmar esta circunstancia.

\section{9.- MODO DE ORGANIZACIÓN Y FUENTES DE PODER}

Si la construcción de residencias elaboradas para los líderes ya constituye de por sí, como decíamos, una manifestación de la desigualdad social institucionalizada (DRENNAN et al., 2010: 45), una mayor profundización en esta misma vía de análisis permite apreciar como este comportamiento también está habitualmente conectado con lo que ha sido definido como modo de organización sociopolítica -o tipo/estrategia de liderazgo- en red/excluyente (network/exclusionary mode o network strategy), propio de algunas sociedades complejas, por oposición al modo corporativo (corporate mode o corporate strategy) (BLANTON et al., 1996). Entre otros aspectos, la estrategia en red implica que los líderes individuales, que asumen en este caso un peso especialmente importante, tratan de monopolizar el poder y de controlar las fuentes de este poder (TRUBITT, 2000: 670). En el caso que aquí planteamos, pensamos que elementos como la gran residencia (Sant Jaume) y el conjunto de circunstancias que la rodean evidencian suficientemente bien no tan solo la existencia de una notable desigualdad social sino, también, el énfasis puesto en un único líder.

Las fuentes de poder que este personaje podría eventualmente haber utilizado para garantizar y perpetuar su posición son diversas. La más evidente en el registro, y probablemente también la más efectiva, vendría representada, como hemos podido comprobar, por los productos de valor obtenidos a través de la red de intercambio a larga distancia establecida con el mundo fenicio -especialmente vino, entre otros-, así como también por aquellos otros productos igualmente valiosos pero obtenidos de un ámbito regional, proporcionados por otras comunidades locales o incluso supralocales más o menos cercanas -posiblemente metales, entre otros-. Todos ellos, aunque especialmente los primeros, podrían ser utilizados, especialmente en el ámbito de la propia comunidad supralocal -aunque no de forma exclusiva-, en una economía de tipo político mediante el desarrollo de una dinámica redistributiva -quizás concretada, al menos parcialmente, en el marco de la realización de banquetes, de tipología diversa- para alcanzar, consolidar y reproducir posiciones de privilegio. De hecho, uno de los aspectos que caracteriza el modo de organización en red/excluyente es que la preeminencia es el resultado del desarrollo y mantenimiento de relaciones de intercambio de carácter individualista, establecidas principalmente fuera del grupo local: la participación en redes extralocales y el subsiguiente acceso diferencial a prestigiosas alianzas matrimoniales, bienes exóticos y/o conocimientos especializados (BLANTON et al., 1996: 5).

Otra fuente de poder podría localizarse en una eventual producción propia de determinados bienes de prestigio, si contemplamos la hipótesis -sustentada, por ahora, en indicios tan solo incipientes- de la existencia de un/os artesano/s especializado/s en la producción de objetos de bronce y/o de hierro residiendo, o al menos trabajando, en Sant Jaume. No resulta tampoco nada despreciable la posibilidad que desde Sant Jaume se gestionara, también con una intención política, una parte del excedente productivo agrario de la comunidad supralocal. Recordemos que buena parte de los pisos superiores de los ámbitos estudiados hasta ahora en el yacimiento han sido interpretados como almacenes, en función de su contenido. En otro orden de cosas, hemos apuntado ya la posibilidad de que, en determinadas circunstancias, el propio edificio de Sant Jaume se acabara convirtiendo a partir de un determinado momento en una nueva fuente de poder, que se sumara a las ya existentes con anterioridad, ayudando a incrementar la fortaleza estructural del sistema. En último término, el conflicto podría haber sido utilizado también como una fuente de poder, vista la importante inversión de tiempo y esfuerzo realizada para fortificar Sant Jaume, los numerosos elementos singulares que caracterizan esta fortificación y el resto de indicios relacionados con las armas y la violencia.

En definitiva, entendemos que en el caso del CSJ, que a la luz de los datos apuntados debe interpretarse como un modelo de jefatura, el liderazgo se identifica especialmente con un individuo en particular y con la utilización que este hace de la riqueza -principalmente de los productos y bienes manufacturados de origen fenicio, pero también de determinados recursos obtenidos de la órbita local, supralocal y regional-, de su residencia, del conflicto y de los símbolos de prestigio para mantener y reafirmar su posición. Todo apunta a que uno de los mecanismos básicos que utilizó para fortalecer y hacer perdurar el sistema fue el establecimiento de una importante dinámica redistributiva, posiblemente de carácter diverso en función del agente a que iba dirigida. Su habilidad principal, con todo, reside en haber sido capaz de establecer en los momentos iniciales -y mantener posteriormente- unos acuerdos firmes que garantizaran unas relaciones hegemónicas y privilegiadas tanto con los agentes fenicios -circunstancia que le proporcionaba el imprescindible acceso exclusivo al comercio de larga distancia, a través del cual llegaban estos productos de valor exóticos- como con otras comunidades indígenas locales y/o supralocales más o menos cercanas 
-que habrían proporcionado las igualmente necesarias materias primeras anheladas por los fenicios-. Gestionando con habilidad la información de que disponía y los contactos que estableció, rasgos habitualmente identificados en momentos de surgimiento de jefaturas (CREAMER \& HASS, 1985), este personaje pudo generar esta estrategia de red como base de una economía política (BLANTON et al., 1996), mediante la cual, utilizando convenientemente los bienes a los cuales tenía un acceso exclusivo, alcanzó una posición de preeminencia sociopolítica a una escala local/supralocal (CSJ) y, hasta cierto punto y con matices, posiblemente también a una escala regional.

\section{0.- CONCLUSIONES}

Hemos analizado una agrupación de asentamientos a la luz de algunos de los parámetros que Drennan y Peterson (2011) proponen para determinar si una serie de evidencias permiten clasificar el modelo de organización sociopolítica propio de una sociedad, tanto del pasado como del presente, como una jefatura. El estudio de esta serie de aspectos sugiere, para el caso estudiado, la existencia de un notable grado de interrelación y jerarquización entre los asentamientos. Constatamos la existencia de una comunidad de tipo supralocal, fundamentada en una jerarquización social y una desigualdad institucionalizada. El grado de centralización política del sistema es alto, concentrando el núcleo de Sant Jaume una importante serie de prerrogativas en relación a determinadas actividades de tipo político y económico, esenciales tanto para la comunidad como para la propia reproducción del sistema. El territorio político controlado de manera directa desde este asentamiento incluiría las áreas inmediatas a la comunidad supralocal conformada por él y por el resto de los asentamientos estudiados, teniendo una extensión pequeña que, quizás, abastaría un centenar de $\mathrm{km}^{2}$. Por lo que respecta a la organización sociopolítica del conjunto y a la interrelación que mantienen los núcleos entre sí, se trata del tipo de agrupación que otros autores han denominado entidades políticas ("polities") o distritos ("districts") (DRENNAN y PETERSON, 2011: 73). En este marco, cada núcleo cumpliría una función diferenciada y complementaria, circunstancia tan solo intuida con anterioridad y que el presente análisis ha permitido contrastar. Se trata de un sistema de integración sociopolítica de tipo jefatura que por sus características -limitada extensión geográfica y demográfica, existencia de tan solo dos niveles de jerarquización entre los asentamientos que conforman la comunidad supralocal y, aparentemente, definición de tan solo un nivel de cargos políticos de orden superiordebe calificarse de simple (STEPONAITIS, 1978).

La interpretación de Sant Jaume como una gran casa y el resto de circunstancias asociadas permiten proponer, efectivamente, que en ella residiera un personaje que disfrutó de un tipo de liderazgo político adscrito, así como que el grado de complejidad sociopolítica existente contempló, más allá del formato que adoptara su posterior concreción histórica y la evolución de este, que la posición de privile- gio y las relaciones de desigualdad asociadas se pudieran transmitir por herencia. Todo ello permite constatar que la base del sistema reside en un modo de organización sociopolítica en red. El conjunto de estas circunstancias indica la existencia de un linaje que en los momentos iniciales de desarrollo del sistema habría afianzado, al menos de forma mínima, esa posición de privilegio, independientemente, como decimos, de las posteriores vicisitudes históricas. En la génesis y desarrollo inicial de esta entidad política polinuclear que hemos venido a denominar Complejo Sant Jaume debieron confluir múltiples causas, aunque conviene asignar en este proceso un papel preponderante al control y a la gestión directa de relaciones comerciales estables con los navegantes fenicios.

A la luz de lo deducido de ese caso de estudio, podemos afirmar, por lo tanto, que por lo que respecta a la protohistoria del nordeste de la península lbérica las evidencias arqueológicas más antiguas que en estos momentos permiten identificar de manera más clara y detallada un tipo de estatus sociopolítico adscrito y una centralidad política, y que, por lo tanto, nos hablan de los primeros pasos claros hacia la complejidad social, política y económica, nos remiten a la región del Sénia y a un momento datado alrededor de mediados del siglo VII anE, es decir, a los inicios de la primera Edad del Hierro.

La existencia en el área del Sénia de una entidad política de base polinuclear regida por un sistema de jefatura simple representa, por su precocidad y excepcionalidad, una importante novedad en el marco de los estudios sobre la evolución de los sistemas sociales y políticos de las primeras etapas protohistóricas de la península lbérica y, muy especialmente, del área del nordeste. Con todo, tenemos la convicción, de manera similar a lo que opinan otros autores, que no estamos ante una excepción. Creemos que existen en esta misma área del nordeste peninsular otras realidades arqueológicas en cronologías similares -y quizás, incluso, más antiguas- que representan formas de organización sociopolítica que deberían clasificarse también como jefaturas. Y estamos convencidos que la continuidad de los trabajos de investigación permitirá ir corroborando esta circunstancia y definir con precisión los rasgos distintivos de cada caso.

\section{1.- AGRADECIMIENTOS}

Al apoyo económico otorgado al proyecto cuadrienal Ref. 2014/100641 por parte del D. de Cultura de la Generalitat de Catalunya y por los ayuntamientos de Alcanar y de Ulldecona.

\section{BIBLIOGRAFÍA}

ARMADA, X.L., GARCIA i RUBERT, D., MONTERO, I., MORENO, I., RAFEL, N. Y ROVIRA, M. C.

2005 "Minería y metalurgia durante la I Edad del Hierro. Procesos de cambio en el sur de Catalunya". Revista d'Arqueologia de Ponent 15, 133-150. 
ATHENS, J.

1979 El proceso evolutivo en las sociedades complejas y la ocupación del periodo tardío-Cara en los Andes septentrionales del Ecuador. Instituto Otavaleño de Antropología, Otavalo.

BEA, D. Y DILOLI, J.

2005 "Elements de representació durant la primera edat del ferro al curs inferior de l'Ebre: el recinte del Turó del Calvari (Vilalba dels Arcs, Terra Alta)". Revista d'Arqueologia de Ponent 15, 179-198.

BEA, D., DILOLI, J., GARCIA i RUBERT, D., MORENO, I. Y MORET, P.

2012 "Arquitectura de prestigio y aristocracias indígenas". En M.C. BELARTE, J.A. BENAVENTE, L. FATÁS, J. DILOLI, P. MORET \& J. NOGUERA (editores). Actas // Congreso Internacional Iberos del Ebro. Alcañiz. Collecció Documenta 25, ICAC, 51-70.

BLANTON, E., FEINMAN, G.M., KOWALEWSKI, S.A. Y PEREGRINE, P.N.

1996 "A Dual-Processual Theory for the Evolution of Mesoamerican Civilization". Current Anthropology 37 (1), 1-14.

BURILLO, F.

1992-93 "La crisis del ibérico antiguo y su incidencia sobre los Campos de Urnas finales del Bajo Aragón". Bajo Aragónprehistoria 9-10: 215-236.

CARNEIRO, R.L.

1981 "The Chiefdom: precursor of the state". En D.D. JONES Y R.R. KAUTZ, (editores) The transition to statehood in the new world. New directions in archaeology. Cambridge, 37-79.

2002 "Was the chiefdom a Congelation of Ideas?". Social Evolution \& History 1(1), 80-100.

CELA, X., NOGUERA, J. Y ROVIRA, C.

1999 "Els materials arqueològics del jaciment ibèric del Coll del Moro de Serra d'Almos (Tivissa, Ribera d'Ebre). Collecció del Museu Comarcal Salvador Vilaseca de Reus". Pyrenae 30, 91-121.

CHABAL, P., FEINMAN, G.M. Y SKALNIK, P.

2004 "Beyond states and empires: chiefdoms and informal politics". Social Evolution y History 3(1), 22-40.

CREAMER, W. Y HAAS, J.

1985 "Tribe versus Chiefdom in Lower central America". American Antiquity 50(4), 738-754.

DRENNAN, R. D. Y PETERSON, C. E.

2006 "Patterned variation in prehistoric chiefdoms". Proceedings of the National Academy of Sciences of the United States of America 103(11), 3960-3967.

2011 "Challenges for comparative study of early complex societies". En M. E. SMITH (editor). The comparative Archaeology of Complex Societies. Cambridge, 62-87.

DRENNAN, R.D., PETERSON, C.E. Y FOX, J.R.

2010 "Degrees and kinds of Inequality". En T.D. PRICE Y G.M. FEINMAN (editores), Pathway to Power: New Perspectives on the emergence of Social Inequality. Springer, 45-76.
EARLE, T.

1987 "Chiefdoms in Arcaheological and Ethnohistorical perspective". Annual Review of Anthropology 16, 279-308.

1991 "The evolution of chiefdoms". En T.K. EARLE (editor). Chiefdoms: Power, Economy and Ideology. Cambridge, 1-15.

1994 "Political domination and social evolution". En T. GOLD (editor), Companion Encyclopedia of Anthropology. Humanity. Culture and Social Life, 940-961.

1997 How chiefs come to power. The political economy in prehistory. Stanford.

FEINMAN, G.M.

1991 "Demography, surplus, and inequality: early political formations in highland Mesoamerica". En T.K. EARLE, (editor), Chiefdoms: Power, economy, and ideology, Cambridge, 229-262.

2005 "The Institutionalization of Leadership and Inequality". En V.L. SCARBOROUGH (editor). A Catalyst for ldeas. Anthropological Archaeology and the Legacy of Douglas Schwartz, Santa Fe.

FONT, L., NADAL, J., MORENO, I. Y GARCIA i RUBERT, D.

2014 "Les restes d'origen animal del jaciment del primer ferro de Sant Jaume - Mas d'en Serrà (Alcanar, Montsià). Interpretació del sector 1 a través de l'estudi zooarqueològic". En Actas XV Colloqui Internacional d'Arqueologia de Puigcerdà, Puigcerdà. 595-606.

\section{GARCIA i RUBERT, D.}

2004 "El plantejament urbanístic i defensiu del poblat de la Moleta del Remei (Alcanar, Montsià) durant la primera edat del ferro". Revista d'Arqueologia de Ponent 14, 179-200.

2005 El poblament del primer ferro a les terres del riu Sénia. Els assentaments de la Moleta del Remei, Sant Jaume, la Ferradura i la Cogula durant els segles VII i VI ane. Universidad de Barcelona. Tesis Doctoral inédita.

2009 "Els sistemes de fortificació de la porta d'accés a l'assentament de la primera edat del ferro de Sant Jaume (Alcanar, Montsià)". Revista d'Arqueologia de Ponent 19, 205-229.

2011 "Nuevas aportaciones al estudio de los patrones de asentamiento en el nordeste de la Península Ibérica durante la Primera Edad del Hierro. El caso del Complejo Sant Jaume". Trabajos de Prehistoria 68(2), 331-352.

\section{GARCIA i RUBERT, D., GRACIA, F. Y MORENO, I.}

2004 "L'impacte del fenomen comercial fenici a les terres del Sénia durant el primer Ferro a partir de l'estudi quantitatiu de la ceràmica. El cas del jaciment de Sant Jaume (Alcanar, Montsià)". Arqueomediterrània 8, 191-201.

2006 "Consideracions sobre la complexitat social durant el primer Ferro al nord-est peninsular. Les comunitats del curs inferior del riu Ebre i de les terres del riu Sénia". Arqueomediterrània 9, 201-220.

\section{GARCIA i RUBERT, D. Y MORENO, I.}

2008 "Marcadors socials durant el primer Ferro a Catalunya el País Valencià. Apunts en relació a l'assentament de Sant Jaume (Alcanar, Montsià)". Arqueomediterrània 10, 215-225.

2009 "Un servei de vaixella procedent de l'assentament de la primera edat del ferro de Sant Jaume (Alcanar, Montsià)". Citerior 5, 97-162. 
GIP [GRUP D'INVESTIGACIÓ PREHISTÒRICA]

2003 "Caballos y hierro. El campo frisio y la Fortaleza de Els Vilars d'Arbeca (Lleida, España), siglos VIII-IV ane". En N. ALONSO, E. JUNYENT, A. LAFUENTE Y J.B. LÓPEZ (editores). Chevaux-de-frise i fortificació en la primera edat del ferro europea, Lérida, 233-274.

\section{HENDERSON, H. Y OSTLER, N}

2005 "Muisca settlement organization and chiefly authority at Suta, Valle de Leyva, Colombia: A critical appraisal of native concepts of house for studies of complex societies". Journal of Anthropological Archaeology 24, 148-178.

\section{JOHNSON, G. A.}

1977 "Aspects of Regional Analysis in Archaeology". Annual Review of Anthropology 6, 479-506.

\section{JOHNSON, A. W. Y EARLE, T.}

2000 The evolution of human societies. From Foraging Group to Agrarian State, Stanford.

LÓPEZ, J.

2007 "Sociedad y economía durante el Bronce Final y la Primera edad del Hierro en el noreste peninsular: una aproximación a partir de las evidencias arqueológicas". Trabajos de Prehistoria 64(1), 99-120.

\section{MARTÍN, A.}

1995 "Formació i desenvolupament de la cultura ibèrica a la zona nord-est de Catalunya". Actas X Colloqui Internacional d'Arqueologia de Puigcerdà, Puigcerdá, 423-447.

MAYA, J.L.

1993 "En torno al origen del mundo ibérico catalán: problemas de substrato", Laietania 8, 9-19.

MORET, P., BENAVENTE, J.A. Y GORGUES, A.

2006 Iberos del Matarraña. Investigaciones arqueológicas en Valdeltormo, Calaceite, Cretas y La Fresneda (Teruel). AlQannis 11.

NETTING, R

1977 Cultural Ecology. Waveland Press, Meno Park.

\section{OLIVER, A Y GUSI, F}

1995 El Puig de la Nau. Un hábitat fortificado ibérico en el ámbito mediterraneo peninsular. Monografies de Prehistòria i Arqueologia Castellonenques 4, Castellón.

\section{PETERSON, C.E. Y DRENNAN, R.D.}

2005 "Communities, settlements, sites, and surveys: regionalscale analysis of prehistoric human interaction". American Antiquity 70(1), 5-30.

2011 "Patterned variation in regional trajectories of community growth". En M. E. SMITH (editor). The comparative Archaeology of Complex Societies, Cambridge, 88-137.

PETERSON, C.E. Y LU, X.

2013 "Understanding Hongshan period social dynamics". En A. P. UNDERHILL (editora). A Campanion to Chinese Archaeology, 55-80.
PETERSON, C.E., LU, X., DRENNAN, R.D. Y ZHU, D.

2010 "Hongshan chiefly communities in Neolithic northeastern China". Proceedings of the National Academy of Sciences of the United States of America 107 (13), 5756-5761.

\section{PONS, E. Y ESTEBA, Q.}

2000 "La signification sociale des nécropoles à incinération en Catalogne côtière". Monographies d'Archéologie Méditerranéenne $7,103-116$.

\section{QUESADA, F.}

1997 El armamento ibérico. Estudio tipológico, geográfico, funcional, social y simbólico de las armas en la Cultura Ibérica (siglos VI-I aC). Monographies Instrumentum, 3.1 y 3.2, Montagnac.

RAFEL, N.

2006 "Sobre el canvi en la Protohistòria. Un cas d'estudi: la primera edat del ferro com a fonament del món ibèric al Matarranya i l'Algars". Arqueomediterrània 9, 135-144.

RAFEL, N. Y ARMADA, X.L.

2009 "Transformación del hábitat y cambio social en la Catalunya meridional (siglos X a VI ane)". Butlletí Arqueològic V, 49-72.

RENFREW, C.

1976 Before Civilization. Cape, Harmondsworth.

RUIZ ZAPATERO, G.

2001 "Las comunidades del Bronce Final: enterramiento y sociedad en los Campos de Urnas". En M.R. GÁLVEZ (Coord.), La edad del Bronce ¿Primera edad de oro de España? Sociedad, economía e ideología, Barcelona, 257-288.

2004 "Casas y tumbas. Explorando la desigualdad social en el Bronce Final y la Primera Edad del Hierro del NE de la Península Ibérica". Mainake XXVI, 293-330.

SAHLINS, M.

1958 Social Stratification in Polynesia. University of Washington Press, Seattle.

1963 "Poor man, rich man, Big-man, Chief: political types in Melanesia and Polynesia". Comparative studies in Society and History 5(3), 285-303.

SANMARTÍ, J.

2004 "From local groups to early states: the development of complexity in protohistoric Catalonia". Pyrenae 35 (1), 7-41.

2009 "From the archaic states to Romanization: a historical and evolutionary perspective on the Iberians". Catalan Historical Review 2, 9-32.

2010 "Demografía y cambio socio-cultural: el caso de la lberia septentrional". Arqueología Espacial 28, 91-108.

SANMARTÍ, J., ASENSIO, D., BELARTE, C. Y NOGUERA, J.

2009 "Comerç colonial, comensalitat i canvi social a la protohistòria de Catalunya". Citerior 5, 219-238.

SARDÀ, S.

2008 "Servir el vino. Algunas observaciones sobre la adopción del oinochoe en el curso inferior del Ebro (s. VII-VI aC)". Trabajos de Prehistoria 65 (2), 95-115. 
2009 "Arqueologia del banquet. Ritualització, semiotica dels aliments i anàlisi contextual". Citerior 5, 15-56.

2010a Pràctiques de consum ritual al curs inferior de l'Ebre (s. VII-VI anE). Comensalitat, ideologia i canvi social. Tesis Doctoral, Universidad Rovira i Virgili: http://www.tdx.cat/TDX-1013110161238 .

2010 b "El giro comensal. Nuevos temas y nuevos enfoques en la protohistoria peninsular". Herakleion 3, 37-65.

\section{SERVICE, E.R.}

1962 Primitive Social Organization. An Evolutionary perspective, Random House, Nueva York.

\section{SIKLÓSI, Zs.}

2004 "Prestige goods in the neolithic of the carpathian basin. Material manifestations of social differentiation". Acta Archaeologica Academiae Scientiarum Hungaricae 55(1-2), 1-62.
SPENCER, J.

1990 "On the tempo and mode of state formation: neoevolutionism reconsidered". Journal of Anthropological Archaeology $9,1-30$.

\section{STEPONAITIS, $\mathrm{V}$.}

1978 "Location Theory and Complex Chiefdoms: A Mississippian example". En B. D. SMITH (editor), Mississippian Settlement patterns, Nueva York, 417-453.

TRUBITT, M.B.D.

2000 "Mound building and prestige goods exchange: changing strategies in the Cahokia Chiefdom". American Antiquity 65-4, 669-690. 
